\title{
Effect of Orientational Specificity of Complexation on the Behavior of Supramolecular Polymers: Theory and Simulation
}

\author{
Matthew C. Hagy, Chun-Chung Chen, and Elena E. Dormidontova* \\ Department of Macromolecular Science and Engineering, Case Western Reserve University, \\ Cleveland, Ohio 44106
}

Received September 15, 2006; Revised Manuscript Received January 19, 2007

\begin{abstract}
Using Monte Carlo simulations we study the association of flexible oligomers terminated by a donor and an acceptor group capable of orientationally specific reversible bonding. On the basis of simulation results, we have obtained equilibrium constants for chain growth and ring closure. These constants were employed in an analytical model, which reproduces the large-scale simulation results very well. We also propose an analytical approach which can be used to analyze experimental data or make predictions of molecular weight, chain/ring distributions, etc., which are hard to obtain experimentally. Our simulation and analytical results show that an increase of orientational specificity of reversible bonding decreases the degree of association and molecular weight and leads to the suppression of small rings. As a result the ring-chain crossover concentration (i.e., the concentration at which the number of reversible bonds in chains and rings coincide) decreases and exhibits a maximum as a function of oligomer length $N$. With a decrease in the energy of reversible association or increase in temperature the ring-chain crossover shifts to lower concentrations and molecular weight either systematically decreases if the system is in the chain-dominated regime (high concentrations) or increases and exhibit a maximum if the system is in ring-dominated regime (low concentrations). Higher orientational specificity of association in combination with a short spacer length ensures a larger value of the molecular weight at its maximum which is reached at lower temperatures and higher oligomer concentrations. These results are supported by recent experimental observations and can be explained based on the oligomer redistribution between chains and rings near the ring-chain crossover.
\end{abstract}

\section{Introduction}

Supramolecular polymers, a new class of self-assembling polymeric materials which employs different types of association motifs (metal-ligand coordination bonds, (self)complementary hydrogen bonded arrays, biorecognition-based complexes), have been introduced by chemists during recent decades and have become a center of growing attention among polymer scientists. ${ }^{1-6}$ The self-healing nature and responsiveness of these polymers to temperature, external fields, etc. implies a large range of potential applications in the general area of "smart materials" or in devices that effect energy, electron or ion exchange.

Taking into account that noncovalent associations is the central element of the "nature" of supramolecular polymers, their properties are ruled by the principles of self-assembly to a much larger extent than for traditional chemically bonded polymers. For instance, depending on the association motifs the supramolecular polymers may have preference for formation of complexes of a particular size/architecture. Also the orientational specificity for end group association may vary depending on the type of association. Physically this implies that the sections of the self-assembled polymer chains adjacent to associated groups have to keep some particular orientation with respect to each other to maintain the association and thereby become effectively more rigid. To some extent the effects of rigidity are not new for condensation polymerization theory - different analytical approaches ${ }^{7-12}$ and simulation techniques ${ }^{12-14}$ were employed to study effects of rigidity on polymerization of chains and especially rings. However in all these approaches rigidity is either considered to be the same for all bonds ${ }^{13-15}$ or averaged over the whole polymer. 9,10 This effect can also be incorporated within the equilibrium constants, which can be different for each

\footnotetext{
* Corresponding author. E-mail: eed@case.edu.
}

ring size. Supramolecular polymers represent a special case in which a spacer can be flexible, but the orientational specificity of associating groups may enforce local rigidity on the polymer. In the present paper, using Monte Carlo simulations in combination with an analytical approach, we intend to address various aspects of the behavior of head-to-tail associating supramolecular polymers which are influenced by the degree of orientational specificity of the association.

So far the experimental observations concerning self-assembly of reversibly associated polymers have been analyzed in the framework of classical condensation polymerization theory, $7,8,12$ equilibrium ring-opening polymerization model, ${ }^{16,17}$ or more recent models by Cates $^{18}$ and Ercolani. ${ }^{11}$ The model by Ercolani ${ }^{11}$ can readily be applicable for supramolecular polymers, except it requires knowing ring-chain equilibrium constants for a range (up to 30) of smaller size rings plus two more constants for all larger rings and all chains. While solution of the proposed equations is rather straightforward, to obtain so many equilibrium constants from experimental measurements is rather cumbersome, taking into account that even the determination of the molecular weight represents a challenge for such "living" polymers. ${ }^{5,19}$ Another technical problem is that the ring-chain equilibrium constant for larger rings is often theoretically estimated based on Gaussian statistics, 7,11,16,17,20,21 although supramolecular polymers formed by self-assembly of a flexible spacers in a good solvent often behave more like chains with excluded volume than Gaussian chains. Associating monomers with excluded volume have been studied in considerable detail in computer simulations, ${ }^{12,13,15,22,23}$ although in these cases orientational specificity either was not considered ${ }^{22,23}$ or it was applied to all monomers (oligomer size one). ${ }^{13-15}$ Experimentally, there have been collected a range of interesting observations, ${ }^{5,20,24}$ which require a more comprehensive model 


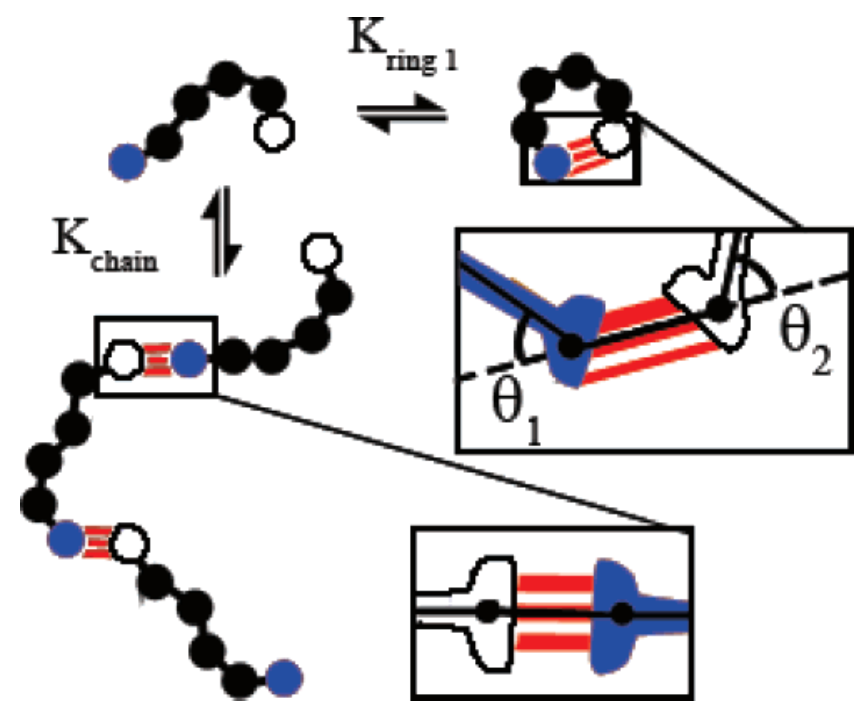

Figure 1. Schematic presentation of reversibly associated polymers (chains and rings) self-assembled from oligomers consisting of flexible spacers terminated by donor and acceptor groups capable of formation of orientationally specific reversible bonds. There are two angles $\theta_{1}$ and $\theta_{2}$ for each complex, which are used to characterize the mutual orientation of the segments adjacent to the donor and acceptor groups.

to gain physical insight into these phenomena. For instance, the experimentally observed increase in viscosity with temperature $^{5,24}$ was either explained based on analogy with ringopening polymerization or using a combination of equilibrium constants as fitting parameters for an analytical model employing Gaussian statistics for rings, which would qualitatively agree with experimental results. ${ }^{20}$ Still a detailed theoretical consideration of this effect for supramolecular polymers, exhibiting some excluded volume effects and more importantly having different rigidity of spacers compared to associating groups, is lacking.

In this paper we propose an analytical approach to account for orientational specificity of supramolecular polymer association without using the assumption of Gaussian statistics. Instead we will use results of Monte Carlo simulations for flexible oligomers of different lengths terminated by a donor and acceptor group capable of orientationally specific reversible bonding with each other (Figure 1). We note that while some experimental systems involve similar architectural concept, others use self-complementary units. ${ }^{4,5,24,25}$ The results presented here for bulk properties of supramolecular polymers are applicable for both cases, with the only correction for selfcomplementary groups is that their equilibrium constants are twice larger (reflecting the fact that the number of associating groups is twice larger for self-complementary units). Varying the orientational specificity and concentration of oligomers we will obtain and analyze the equilibrium constants for ring and chain formation. On the basis of these results, we will propose a simplified analytical model which can be readily used to analyze experimental results and make predictions regarding the degree of association, molecular weight and ring-chain crossover concentration. We will also study the effect of orientational specificity, oligomer length and association energy (temperature) on the ring/chain population, molecular weight dependence and ring-chain crossover concentration. These results will be compared with available experimental data, in particular the comparison will be made between the predicted maximum in the molecular weight temperature dependence and observed maximum in viscosity of hydrogen-bonded supramolecular polymers. ${ }^{5,24}$
This paper is arranged in the following way. In the next two sections, we will briefly discuss our simulation and analytical approaches. Then the results of simulations for the equilibrium constants of chain growth and ring closure via a reversible bond will be presented. This will be followed by the discussion of the simulation results in comparison with an analytical model for the degree of association and ring/chain population. In this section, we will also introduce our simplified model, which can be used to analyze/predict experimental results. We will continue with a discussion of the influence of orientational specificity of association on the ring-chain crossover concentration as a function of oligomer chain length and energy of association (or temperature). We will also discuss the molecular weight dependence on concentration, orientational specificity and temperature, making comparison with experimental results when appropriate. Finally we summarize our main findings in the Conclusions section.

\section{Simulation Methods}

To study the effect of orientational specificity of the association on self-assembly of head-to-tail reversibly associating polymers we applied the bond-fluctuation model (BFM) using Monte Carlo simulations. ${ }^{26,27}$ Each simulation setup consisted of a fixed number $\mathcal{N}$ of identical oligomers. Within the framework of BFM, an oligomer was modeled as a series of $N$ monomers joined by totally flexible covalent bonds. Each oligomer contains at the ends one donor and one acceptor, capable of reversible association with each other. Formation of each reversible bond decreased the system energy by $\Delta E_{\text {assoc }}=8 k T$. To model the orientation specificity of reversible bonding we have considered a weighting factor for formation of a reversible bond for a given angle $\theta, P(\theta)=\exp [-$ $B(1-\cos \theta)$ ], where the rigidity parameter $B$ (defined in units of the Boltzmann constant $k$ ) is assumed to be independent of temperature. ${ }^{28}$ As is seen from Figure 1, there are two such angles to consider for each reversible bond. We note that all permanent chemical bonds within the oligomer were considered to be totally flexible; i.e., there was no entropic penalty for bending any of them. We used a cubic lattice of lateral size $64 a$ or $128 a$, (with $a$ being the unit lattice spacing), with periodic boundaries in all three directions to prevent surface effects. Volume exclusion between monomers and bond length constrains were subject of the standard BFM rules $(l=2 a, \sqrt{5} a, \sqrt{6} a, 3 a, \sqrt{10} a)^{26,27}$ while each monomer occupies an effective volume of $8 a^{3}$.

The system was brought to an equilibrium through a Monte Carlo algorithm consisting of a series of random updates (MC steps). Each MC step consisted of $1 N$ monomer displacement attempts. The reversible bonds were considered to be the same as covalent bonds during such moving attempts. Additional bonding updates (including cleavage of a reversible bond (if any) and formation of a new reversible bond (if any)) were performed following all moving attempts of donors. ${ }^{29}$ If a donor was reversibly bonded prior to the move, this reversible bond will be broken and bonding update will be performed: if there are free acceptors in a bonding distance from the donor, a reversible bond is formed with an available free acceptor with the probability $C P(\theta) \mathrm{e}^{\Delta E_{\text {assoc }} /(k T)}$, or no bonds will be formed with probability $C=\left(1+\sum_{i=1}^{N_{\mathrm{a}}} P(\theta) \mathrm{e}^{\Delta E_{\text {assoc }} d(k T)}\right)^{-1}$ (where $N_{\mathrm{a}}$ is the total number of available free acceptors for this donor). As a result of the bonding update a new reversible bond can be formed or old one restored or no bonds formed at all depending on the corresponding probabilities. We note that this method of bonding update is different from the commonly used Metropolis algorithm but it also satisfies detailed balance. As the new bonding configuration of the system is directly drawn from the Boltzmann probability distribution, the system has a minimal potential barrier for the bonding-unbonding event in this case ${ }^{30}$ resulting in fast equilibration for the reversible bonding. A system was allowed to equilibrate for $2^{22} \mathrm{MC}$ steps and equilibrium data were measured and averaged over subsequent MC steps. In system simulations we 
recorded the average number of species of size $i$ distinguishing between linear chains $C_{i}$ and rings $\mathrm{R}_{i}$. From these data, we calculated the equilibrium molecular weight, degree of association and ring/chain distributions for a system. The same simulation technique was applied to study conformations of a single oligomer (for details, see the Supporting Information), which was used to determine the equilibrium constants for ring and chain formation, as discussed below.

\section{Analytical Model}

As we discussed in our previous paper, ${ }^{29}$ the overall degree of association in head-to-tail reversibly associated system can be described by the following simple equation:

$$
p=\exp \left[\frac{\Delta F_{\text {assoc }}}{k T}\right](1-p)^{2} \frac{v \mathcal{N}}{V}
$$

with $\Delta F_{\text {assoc }}$ being the free energy change for formation of a reversible bond, $p$ is the overall degree of association between a donor and an acceptor groups, $v$ is the reference volume (discussed below) and $V$ is the total volume of the system. This equation works reasonably well at high polymer concentration, but fails to describe correctly the low concentration regime where ring formation becomes important. In this paper, we will consider more general approach which accounts for ring formation. Considering the individual equilibria between chain growth and cyclization, one can write the mass balance equations as follows: ${ }^{11}$

$$
\begin{gathered}
\mathrm{O}]=\sum_{i=1}^{\infty} i\left[\mathrm{C}_{i}\right]+\sum_{i=1}^{\infty} i\left[\mathrm{R}_{i}\right] \\
{\left[\mathrm{C}_{i}\right]=\left[\mathrm{R}_{1}\right]^{i} K_{\text {chain }}^{i-1} K_{\text {ring }, 1}^{-i} ;\left[\mathrm{R}_{i}\right]=\left[\mathrm{C}_{i}\right] K_{\text {ring }, i}} \\
{\left[\mathrm{C}_{i-1}\right]+\left[\mathrm{C}_{1}\right] \stackrel{K_{\text {chain }}}{\rightleftharpoons}\left[\mathrm{C}_{i}\right] ;\left[\mathrm{C}_{i}\right] \stackrel{K_{\text {ring, } i}}{\rightleftharpoons}\left[\mathrm{R}_{\mathrm{i}}\right]}
\end{gathered}
$$

where $\left[\mathrm{C}_{i}\right]$ and $\left[\mathrm{R}_{i}\right]$ are the equilibrium number density of rings and chains respectively and $[\mathrm{O}]=\mathscr{M} V \equiv n$ is the initial number density of oligomers. The equilibrium constants for chain

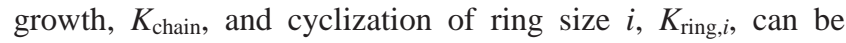
calculated from the simulation results, as discussed below. The mass balance equations can then be numerically solved to obtain the equilibrium distribution of rings and chains and other properties of supramolecular polymers. We note that eqs 3 and 4 essentially represent "chemical equilibrium" between rings and chains of different sizes, where any concentration or chain length dependence of association is hidden in the equilibrium constants as well as any other factors which may influence the association process such as stacking interactions between associated complexes, volume interactions between the associating groups and spacers, etc. Some of these effects, such as the chain-length dependence of ring equilibrium constant, were accounted for in our simulations and described in the analytic model, discussed below. Some others, such as concentration dependence of the equilibrium constants, were found to be rather small in our simulations (at least in the concentration range considered). The importance of other effects still remains a subject of our future studies, but we expect them to be less important at relatively low concentrations.

We note that a somewhat different mass balance equation was considered by Petschek et al. ${ }^{16}$ and by Corrales and Wheeler ${ }^{17}$ where three equilibrium constants for activation, chain and ring growth were considered. Since for supramolecular polymers there is no activation step for the associating groups to interact, there is no real activation constant in our case, although the inverse of the equilibrium constant for the smallest ring, $K_{\text {ring,1 }}$ would have a somewhat analogous meaning. Following Ercolani, we are considering the equilibrium constant to be different for all rings including a monomolecular ring, whereas in ring-opening equilibrium polymerization models 16,17 the ring growth constant is connected with chain constant via a simple relation including a power-law dependence on ring size. The latter is the result of the assumed Gaussian statistics, which inherently does not include any rigidity effects. In our approach, we do not make these assumptions. Additionally the bonds between monomers of a spacer are different from the reversible bonds in both their reversibility and rigidity. We will discuss below in what limit our model follows the approximations of equilibrium living polymerization theory. ${ }^{16,17}$

Equilibrium Constant for Chain Growth. In order to form a reversible bond between oligomers in a linear chain the donor and acceptor groups should be within a bonding distance, i.e., an acceptor should be located within the volume $v_{\text {assoc }}$ of the donor. If $v$ is a reference volume, then the entropic loss for having donor and acceptor groups in vicinity of each other is $\Delta S_{\text {ends }}=-\ln v_{\text {assoc }} v$, where $v_{\text {assoc }}$ is an association volume (i.e., the donor and acceptor groups should be inside this volume for the association to occur). ${ }^{29}$ We note that $\Delta S_{\text {ends }}$ (and all other entropies mentioned in the paper) is actually the entropic loss in units of the Boltzmann constant $k$. In the case of no orientational specificity $(B=0)$, any mutual location of donor and acceptor inside the reference volume should allow reversible bond formation, i.e., $\Delta S_{\text {ends }}=0$ and $v_{\text {assoc }}=v$. In the BFM, $v$ is approximately constant and independent of oligomer length and monomer concentration. We approximated this value by fitting the simulation results for the chain degree of association (for different concentrations and oligomer lengths) in the case of flexible complexes $(B=0)$. The best fit of the data (not shown here) corresponds to $v \approx 54 a^{3}$.

In the case of directionally specific reversible bonds $(B>$ 0 ), there will be an additional entropic loss as the donor and acceptor groups should be not only within a bonding distance from each other, but also within the range of allowable angles $0 \leq \theta \leq \theta_{\max }$ imposed by the conformational limitations. The corresponding entropic loss associated with the angle specificity is

$$
\exp \left(\Delta S_{\text {bend }}\right)=-\frac{\int_{0}^{\theta_{\max }} P(\theta) \sin \theta \mathrm{d} \theta}{1-\cos \theta_{\max }}
$$

Upon integration, we arrive at

$$
\Delta S_{\text {bend }}=-\ln \left|\frac{\exp \left[-B\left(1-\cos \theta_{\max }\right)\right]-1}{-B\left(1-\cos \theta_{\max }\right)}\right|
$$

The value of $\cos \theta_{\max } \simeq-0.5$ was determined as a best fit to the simulation results for entropic loss plotted vs the rigidity constant, $B$, shown in Figure 2.

The simulation data points for $\Delta S_{\text {bend }}$ were obtained from comparison of the equilibrium constants $K_{\text {chain }}$ for chain growth for orientationally specific bonding (see Supporting Information) with that for the flexible complexation $(B=0)$.

As is seen from Figure 2, $\Delta S_{\text {bend }}$ noticeably increases with an increase of orientational specificity of association as the penalty for deviation from linearity of a reversible bond grows with $B$ (as described in the weighting factor, $P(\theta)) . \Delta S_{\text {bend }}$ does not change appreciably with oligomer length or concentration as the data for two oligomer lengths $(N=4$ and $N=16)$ and two different concentrations $\left(n=2^{-9} a^{-3} \approx 1.953 \times 10^{-3} a^{-3}\right.$ 


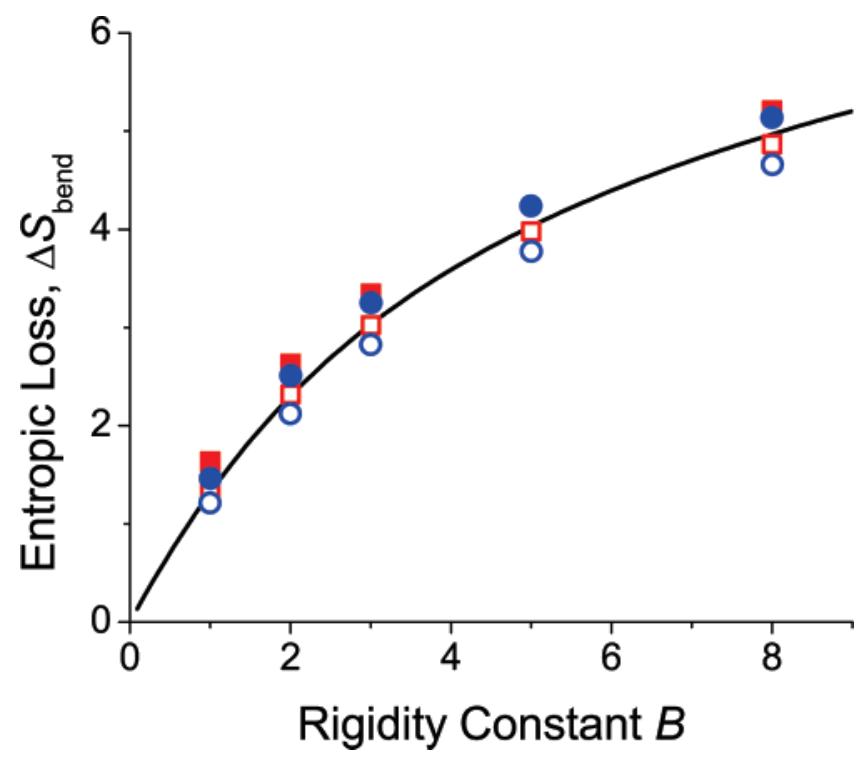

Figure 2. Entropic loss of chain growth $\Delta S_{\text {bend }}$ as a function of the rigidity constant $B$ characterizing the orientational specificity of reversible bonding. Simulation results obtained for oligomer length $N$ $=4$ are shown as squares and $N=16$ as circles. The results for oligomer number density $n \approx 0.0019 a^{-3}$ are depicted as solid symbols and for $n \approx 6.1 \times 10^{-5} a^{-3}$ as open symbols. The curve is the solution of eq 6 .

and $n=2^{-14} a^{-3} \approx 6.1 \times 10^{-5} a^{-3}$ ) follow each other closely. We note that with an increase of concentration the probability of finding an acceptor located at a bonding distance and within the binding angle of a donor may change, so that $\Delta S_{\text {bend }}$ and the equilibrium constant for chain growth, $K_{\text {chain, may vary }}$ slightly with concentration. However, since this dependence is rather weak, it will be neglected in further calculations.

Summing up different terms determining the chain growth

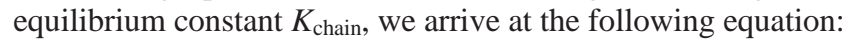

$$
K_{\text {chain }}=v \exp \left(-\Delta S_{\text {bend }}+\frac{\Delta E_{\text {assoc }}}{k T}\right)
$$

Equilibrium Constant for Ring Formation. As with chain growth, to form a ring by a reversible bond, a donor and an acceptor have to be within a bonding distance. The main difference is that the donor and acceptor have to be a part of the same chain, so that the entropic loss for ring closure will be a function of chain length. To determine the probability of finding ends of a chain within a bonding distance, $P_{\text {closure, }}$ we performed simulations of a single oligomer. This probability is shown in Figure 3 as a function of oligomer length. As is seen $P_{\text {closure }}$ follows a power-law dependence $P_{\text {closure }}(N) \simeq D N^{-\alpha}$ with $D \approx 1.25$ and exponent $\alpha \approx 2$, which is somewhat larger than $3 / 2$ for a 3 -d Gaussian chain. ${ }^{7}$ This difference is not surprising as our simulations account for excluded volume effects, which would increase the value of $\alpha$ to about 1.92-1.97 expected for the good solvent case, ${ }^{31}$ which is very close to what we see. Using the obtained power law dependence, we can determine the entropic loss of ring closure $\Delta S_{\text {closure }}$ as

$$
\Delta S_{\text {closure }, 1}(N)=-\ln P_{\text {closure }}(N) \sim \alpha \ln N
$$

In the case of directionally specific reversible bonds $(B>0)$, there will be an additional entropic loss for ring formation as the range of possible angles between the segments adjacent to the donor and acceptor groups will be limited by the conformation of the rest of the chain having its ends at a bonding distance. Naturally the angle limitation and therefore the corresponding

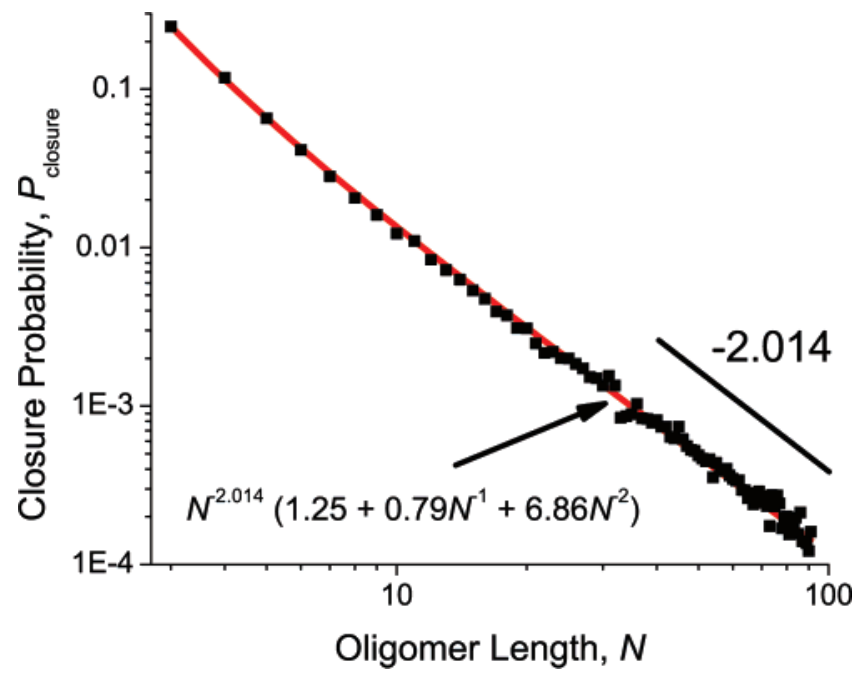

Figure 3. Ring closure probability determined from simulations of a single flexible $(B=0)$ oligomer as a function of oligomer length $N$. The best power law fit of the data $P_{\text {closure }}=N^{-2.014}\left(1.25+0.79 N^{-1}+\right.$ $6.86 N^{-2}$ ) is shown as a solid curve. The slope of the curve for larger $N$ values is -2.014 .

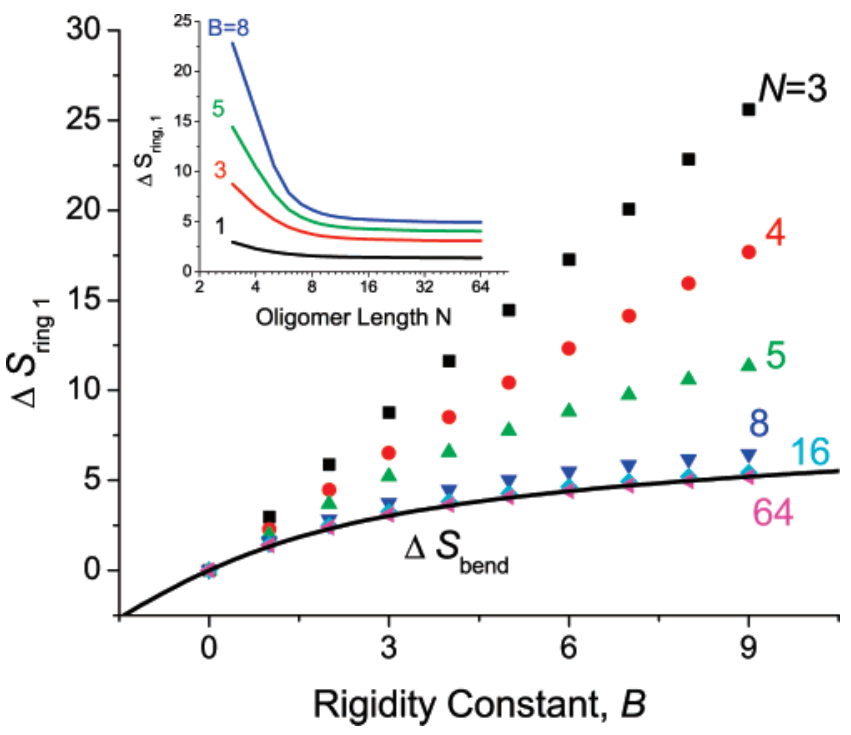

Figure 4. Entropic loss of the reversible bond formation in a monomolecular ring $\Delta S_{\text {ring, } 1}$ as a function of the rigidity parameter $B$ (see text). The main figure shows $\Delta S_{\text {ring, } 1}$ for monomolecular rings of different oligomer length ( $N=3-64$ from top to bottom). The solid curve corresponds to the entropic loss for chain growth $\Delta S_{\text {bend }}$ (the same as in Figure 2). The inset shows the dependence of $\Delta S_{\text {ring,1 }}$ on oligomer length $N$ for different rigidity constants $B$.

entropic loss $\Delta S_{\text {ring,1 }}$ will be the largest for short chains/ oligomers. This entropic loss for small rings was determined from the simulation of a single ring. In this simulation the donor-acceptor association was irreversible and there was no orientational specificity $(B=0)$; i.e., it was indistinguishable from the internal covalent bonds of an oligomer. We collected the distribution of angle pairs associated with the donoracceptor bond for a range of oligomer lengths $N$. The entropic loss for the formation of orientationally specific reversible bond $\Delta S_{\text {ring, } 1}(N)$ could then be calculated by integration over the angle distribution weighted by the factor $P(\theta)$. Figure 4 shows $\Delta S_{\text {ring, } 1}$ calculated using this approach as a function of the reversible bond rigidity constant $B$ for constant oligomer length $N$ (main plot) and as a function of $N$ for constant $B$ (inset). As is seen, for all oligomer lengths $\Delta S_{\text {ring, } 1}$ increases with an increase of orientational specificity and decreases with an increase of oligomer length, as expected. For very small rings (formed from 
short oligomers) we observe a nearly linear dependence of the entropic loss on $B$. This is the result of the strong ring geometry constraints favoring a particular angle of bonding. We note that the absolute value of the preferable bonding angle is dependent on our simulation setup (lattice type, allowed bond lengths, etc.). For real experimental systems there also will be some preferable angle for small ring closure, which is dictated by the individual chemical architecture of the spacer and associating groups. Therefore, the results presented below are expected to reproduce the general trend correctly, without attempting a quantitative fit of the association behavior of any particular system. For larger oligomer lengths the dependence of $\Delta S_{\text {ring, } 1}$ on $B$ becomes more complex as the range of available angles for bonding expands. In the limit of long oligomers the entropic loss for the formation of orientationally specific reversible bonds closing the ring tend to the same limit as that for chain growth, i.e.

$$
\begin{aligned}
\left.\Delta S_{\text {ring, }, 1}\right|_{N>N_{\max }} \simeq \Delta S_{\text {bend }} & = \\
- & \ln \left|\frac{\exp \left[-B\left(1-\cos \theta_{\text {max }}\right)\right]-1}{-B\left(1-\cos \theta_{\max }\right)}\right|
\end{aligned}
$$

As is seen from Figure 4 (and Table S1 presented in Supporting Information) the limit of long oligomers is reached approximately at $N_{\max } \approx 15$ according to the main plot in Figure 4 or even smaller $N_{\max } \approx 10$ for smaller rigidities $B$ (inset of Figure 4). For all oligomers of larger length eq 9 can be used for the entropy of orientationally specific ring closure by a reversible bond.

Besides monomolecular rings there will also be rings of larger size containing $i=2,3, \ldots$, oligomers. In general a similar approach as described above (i.e., using single chain or ring simulations) can be applied to find $\Delta S_{\text {closure }, i}$ and $\Delta S_{\text {ring, } i}$. Analysis of our results (such as ring distribution presented in the Supporting Information, degree of association or molecular weight discussed in the next section) shows that this may not be necessary as the entropic loss for a ring of size $i$ (i.e., containing $i$ oligomers of length $N$ ) can be well estimated using the corresponding entropic loss for monomolecular ring of appropriate size, $i N$, i.e.

$$
\begin{aligned}
\Delta S_{\text {closure }, i}(N) & \simeq \Delta S_{\text {closure }, 1}(i N) \\
\Delta S_{\text {ring }, i}(N) & \simeq \Delta S_{\text {ring }, 1}(i N)
\end{aligned}
$$

Combining different factors influencing formation of rings, we arrive at the following equation for the cyclization equilibrium constant of ring size $i$

$$
\begin{aligned}
K_{\text {ring }, i}(N) & =\frac{1}{i} \exp \left[-\Delta S_{\text {closure }, i}(N)-\Delta S_{\text {ring }, i}(N)+\frac{\Delta E_{\text {assoc }}}{k T}\right] \\
& \simeq \frac{1}{i} \exp \left[-\Delta S_{\text {closure }, 1}(i N)-\Delta S_{\text {ring }, 1}(i N)+\frac{\Delta E_{\text {assoc }}}{k T}\right]
\end{aligned}
$$

The factor $i^{-1}$ preceding the exponent reflects the fact that there are $i$ reversible complexes in the ring where the ring can be broken. ${ }^{32,33}$ In contrast to the chain growth equilibrium constant $K_{\text {chain }}$, the cyclization equilibrium constant $K_{\text {ring, } i}(N)$ is a strong function of oligomer length (at least for small rings). We note that distribution of cyclization equilibrium constants $K_{\text {ring, } i}$ obtained from our simulations shows rather similar trend as was experimentally observed for polycycloolefins ${ }^{34}$ including the decrease of the association constants for small rings with a decrease of $N$ and $K_{\text {ring, } i} \simeq i^{-3.2}$ dependence for larger rings and for corresponding ring concentrations. ${ }^{11,22,23,34}$ In general, $K_{\text {ring, } i}$ may also slightly depend on polymer concentration, but we expect this dependence to be even weaker than that for $K_{\text {chain }}$ as rings are not the most common species at large concentration, so this dependence will be neglected.

Equation for Chain Degree of Association $\boldsymbol{x}$. Knowing the equilibrium constants for ring formation and chain growth, and using the Jacobson-Stockmayer relations for the concentration of chains of size $i$ (i.e., containing $i$ oligomers), ${ }^{7}$ one can rewrite the mass balance equation in the following form (similar to that derived by Ercolani ${ }^{11}$ )

$$
\frac{1}{K_{\text {chain }}} \sum_{i=1}^{\infty} i x^{i} K_{\text {ring }, i}+\frac{1}{K_{\text {chain }}} \sum_{i=1}^{\infty} i x^{i}=[\mathrm{O}]
$$

where the chain degree of association $x$ is connected with the concentration of monomolecular rings and the equilibrium constants for ring formation and chain growth via the equation

$$
x=\frac{\left[\mathrm{R}_{1}\right] K_{\text {chain }}}{K_{\text {ring }, 1}}
$$

Performing the summation of the terms describing the chain contribution and subdividing the ring contribution into the part responsible for small $\left(N \leq N_{\max }\right)$ and large rings $\left(N>N_{\max }\right)$, eq 13 can be rewritten in the following form:

$$
\begin{aligned}
\frac{1}{K_{\text {chain }}} & \sum_{i=1}^{i \leq N_{\max } / N} i x^{i} K_{\text {ring }, i}+ \\
& \frac{1}{K_{\text {chain }}} \sum_{i>N_{\max } / N}^{\infty} i x^{i} K_{\text {ring }, i}^{*}+\frac{1}{K_{\text {chain }}} \frac{x}{(1-x)^{2}}=[\mathrm{O}]
\end{aligned}
$$

where $K_{\text {ring, } i}$ is defined by eq 12 , with $\Delta S_{\text {ring, }, 1}(i N)$ being obtained from single ring simulations (as shown in Figure 4 and Table $\mathrm{S} 1$ presented in Supporting Information), and

$$
\begin{aligned}
& K_{\text {ring }, i}^{*}(N)=\frac{1}{i} D(i N)^{-\alpha} \exp \left(-\Delta S_{\text {bend }}+\frac{\Delta E_{\text {assoc }}}{k T}\right)= \\
& \frac{1}{i} D(i N)^{-\alpha} \frac{K_{\text {chain }}}{v}
\end{aligned}
$$

where $D \approx 1.25$ (see Figure 3 ). We note that the equilibrium constant for larger rings $K_{\text {ring, } i}^{*}(N)$ (eq 16) recovers the approximation used in equilibrium ring-opening polymerization, ${ }^{16,17}$ except that the exponent of the power law dependence on ring size is somewhat different due to the excluded volume effects considered here and the numerical prefactor is also much larger reflecting larger size of our associating units: oligomers vs monomers, as were considered in equilibrium ring-opening polymerization. ${ }^{16,17}$ With these exceptions, our model will be analogous to the equilibrium ring-opening polymerization theory in the limit of long oligomers.

The modified (eq 15) or original (eq 13) mass balance equation can be solved for the chain degree of association $x$ (which was done numerically using a binary search algorithm under the assumption of a single real root in the range $0<x<$ 1). We note that in practically all cases considered the contribution of rings is limited to a relatively small range of ring sizes, $i \lesssim 15$, so often the higher order terms in summation over the rings (eq 15) can be omitted without compromising the quality of predictions (see results presented below). Moreover, in the next section, we will discuss the simplified version of the equation which while being somewhat less accurate in some cases, can be more useful in practical applications. Knowing $x$, the complete chain and ring distribution can be 

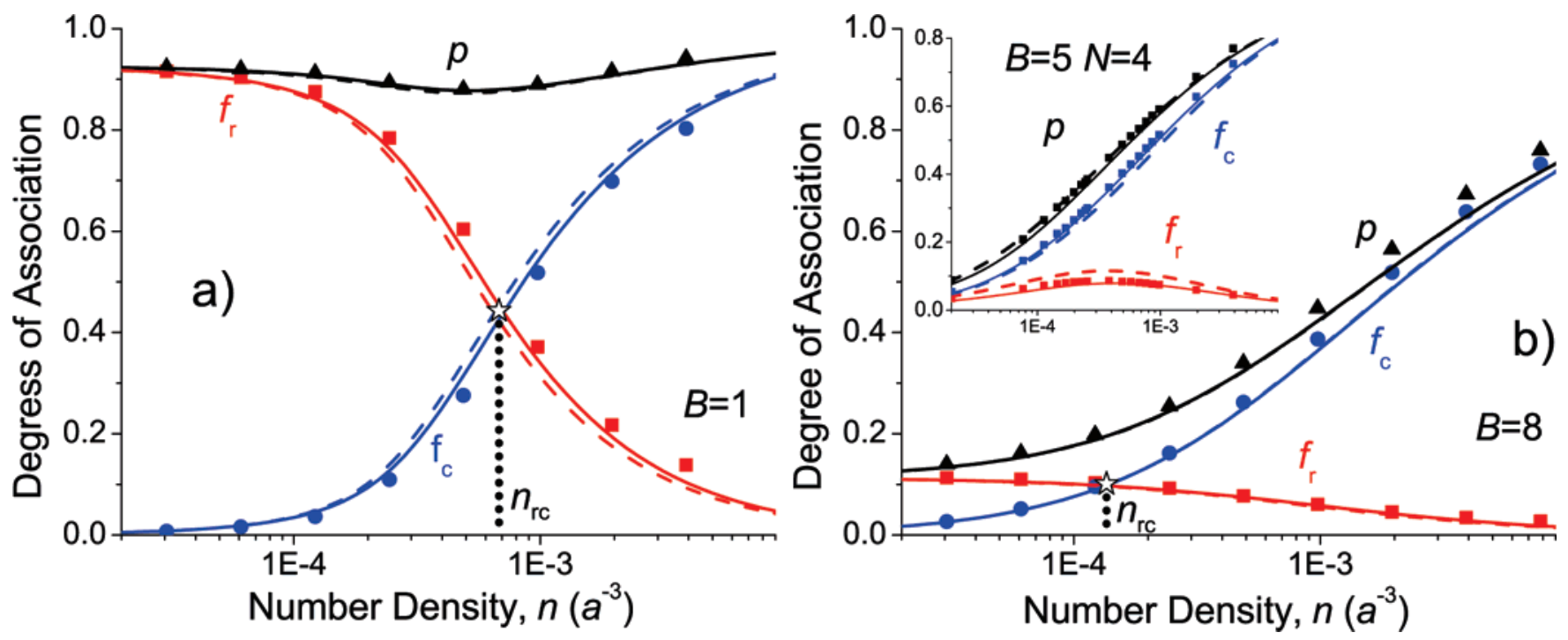

Figure 5. The total degree of association $p$ and the fraction of reversible bonds in rings $f_{\mathrm{r}}$ and chains $f_{\mathrm{c}}$, as a function of number density for oligomers for $N=8, B=1$ and 8 (a and b, respectively) and $N=4, B=5$ (b, inset). Simulation results are shown as symbols, while the curves are the solutions of the complete (eq 15, solid lines) and approximate (eq 23, dashed lines) mass balance equation. The solution of the approximate equation is practically indistinguishable from the solution of the complete equation for $N=8, B=8$ (b, main plot). Vertical dotted lines with stars indicate the critical number density $n_{\mathrm{rc}}$ corresponding to the ring-chain crossover.

recovered and other macroscopic properties such as the overall degree of association, molecular weight, etc. can be obtained. We will discuss these results in the following section.

\section{Results and Discussion}

Concentration Dependence of the Overall Degree of Association $p$. Solving the eq 13 (or eq 15) for $x$ we can obtain the ring $\left[\mathrm{R}_{i}\right]$ and chain $\left[\mathrm{C}_{i}\right]$ distributions and calculate the degree of association as follows

$$
p=f_{\mathrm{r}}+f_{\mathrm{c}} \equiv \frac{1}{[\mathrm{O}]} \sum_{i=1}^{\infty} i\left[\mathrm{R}_{i}\right]+(i-1)\left[\mathrm{C}_{i}\right]
$$

The first term in the equation accounts for the fraction of reversible bonds in rings: $f_{\mathrm{r}} \equiv \sum_{i=1}^{\infty} i\left[\mathrm{R}_{i}\right] /[\mathrm{O}]$. This value together with the direct simulation results are shown in Figure 5 (as solid curve and squares, respectively) as a function of oligomer number density, $n=\mathcal{M} V$, for oligomer length $N=8$ and rigidity parameter $B=1$ (Figure 5a) and $B=8$ (Figure 5 b). As is seen the analytical prediction obtained using eq 15 agrees very well with the simulation results in both cases (as well as for all other $B$ values considered). In the zero concentration limit the system properties are defined by the behavior of a single oligomer, and as a result the overall degree of association coincides with that for monomolecular rings in this limit. The larger the orientational specificity of reversible bonding, the higher the penalty for formation of a monomolecular ring via an orientationally unfavorable reversible bond (i.e., larger $\Delta S_{\text {ring, } 1}$ ) and as a result the degree of association is noticeably lower for $B=8$ compared to $B=1$ for the same oligomer length. In both cases the maximum degree of association for rings is reached at the lowest concentration. This is a typical behavior for head-to-tail associating polymers of a sufficient spacer length $N .{ }^{23}$ If a spacer length is rather small and orientational specificity of association is high then formation of monomolecular rings can be considerably suppressed due to the strain inflicted on the oligomer by the angle limitations for ring closure (see the ring distribution in Supporting Information). As a result the maximum degree of association for rings is achieved not at the lowest concentration, but at some higher concentration, when rings of size two (i.e., composed from two oligomers) dominate, i.e., when $K_{\text {ring, } 2}>K_{\text {ring, } 1}$ (as shown in the Appendix). An example of such a situation is shown in the inset of Figure $5 \mathrm{~b}$ for oligomer length $N=4$ and $B=5$.

As the concentration is increased, chains of increasing molecular weight will form in competition with rings of larger sizes. The fraction of reversible bonds in chains is described by the second term in the eq 17: $f_{\mathrm{c}} \equiv \sum_{i=1}^{\infty}(i-1)\left[\mathrm{C}_{i}\right] /[\mathrm{O}]$. Similar to the ring fraction, $f_{\mathrm{c}}$ calculated using the analytical model agrees rather well with the direct simulation results (shown as circles in Figure 5). An increase of concentration favors the formation of intermolecular complexes and as a result the chain degree of association considerably increases whereas the fraction of reversible bonds in rings decreases. For sufficiently large rings the entropic loss for ring closure via an orientationally specific reversible bond $\Delta S_{\text {ring, } i}$ becomes comparable to that for chains $\Delta S_{\text {bend. }}$. However the probability of finding two ends of a long chain separated by a bonding distance is quite small and decreases with ring size $i$ as $(i N)^{-\alpha}$ (i.e., entropy loss, $\Delta S_{\text {closure }, i}$, increases as $\left.\alpha \ln (i N)\right)$. As a result the fraction of large rings and the overall value of $f_{\mathrm{r}}$ remains rather low, so that the overall degree of association is determined predominantly by chains at higher oligomer concentrations. As we discussed in our previous publication, ${ }^{29}$ for this reason eq 1 can be used to describe the overall degree of association at higher oligomer concentrations. Taking into account that for larger polymer concentrations $\Delta F_{\text {assod }} / k T=-\Delta S_{\text {bend }}+\Delta E_{\text {assod }}$ $k T$, eq 1 becomes

$$
p=K_{\text {chain }}(1-p)^{2} \frac{\mathcal{N}}{V}, \text { for large } \frac{\mathcal{N}}{V}
$$

and the overall degree of association should depend only on $K_{\text {chain }} / T V \equiv n K_{\text {chain }}$ (see, e.g., ref 35 ).

The overall degree of association, i.e., $p=f_{\mathrm{r}}+f_{\mathrm{c}}$ is shown in Figure 6 as a function of $n K_{\text {chain }}$ for oligomer length $N=8$ and different $B$ values. As is seen, the degree of association $p$ obtained for different $B$ values coincides at larger polymer concentrations (as long as the product $n K_{\text {chain }}$ is the same) forming a unified curve, as expected by eq 18 . At lower polymer concentrations, where rings dominate, the degree of association 


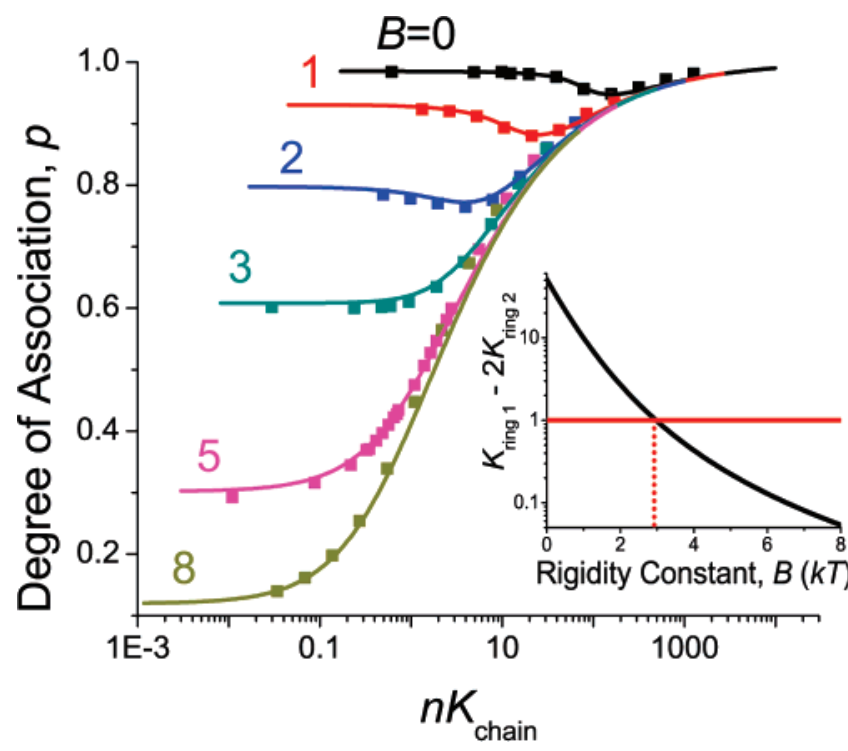

Figure 6. Total degree of association $p$ as a function of $n K_{\text {chain }}$ for $N$ $=8$ and various rigidity constants $B$. Simulation results are shown as symbols, while the curves are the solutions of the complete (eq 15, solid lines) and approximate (eq 23, dashed lines) mass balance equation. The solution of the approximate equation is practically indistinguishable from the solution of the complete equation, except for low rigidity constant $(B=0,1)$. The inset shows $K_{\text {ring, } 1}-2 K_{\text {ring, } 2}$ as a function of the rigidity constant $B$. When $K_{\text {ring, } 1}-2 K_{\text {ring, } 2}>1$, a minimum in the total degree of association is expected.

$p$ noticeably decreases with an increase in $B$. As we discussed above, formation of chains and rings (especially monomolecular rings), is strongly affected by orientational limitations. As a result the overall degree of association for the supramolecular polymers employing complexes of higher orientational specificity will be smaller than that for more flexible associations in both the high and low concentration range (for similar $n$ ). We note that the analytical predictions obtained by solving eq 15 work very well for the whole range of concentrations and $B$ values considered.

Another interesting feature of the concentration dependence shown in Figure 6 is the nonmonotonic behavior observed for the overall degree of association at low orientational specificity of reversible bonding. As is seen for $B=0,1$, and 2 , the degree of association first decreases, passes through a minimum, and then increases with an increase in oligomer density. For higher $B$, the degree of association monotonically increases without any extremum. We found that the main factor determining whether the overall degree of association will follow one or the other pattern is the ring degree of association at low concentration. If it is sufficiently large $\left(f_{\mathrm{r}} \gtrsim 0.6\right.$ for Gaussian chains, as discussed in the Appendix), then most of the oligomers will be present in the form of monomolecular rings at low concentrations which will slow down the formation of larger chains and rings. As a result the overall degree of association will first decrease followed by an increase when the formation of chains starts to dominate at larger concentration. An increase in the orientational specificity of reversible bonding diminishes the monomolecular ring population. As a result, having a large number of nonassociated oligomers chain growth (or formation of rings of larger size) occurs earlier and the overall degree of association increases with concentration. Simple calculations (see the Appendix) based on the consideration of formation of monomolecular rings in comparison with chains and rings containing two oligomers show that a minimum in the concentration dependence of the overall degree of association is expected when

$$
K_{\text {ring }, 1}-2 K_{\text {ring, } 2} \geq 1
$$

Plotting $K_{\text {ring, } 1}-2 K_{\text {ring, } 2}$ as a function of $B$ for oligomer length $N=8$ (Figure 6 , inset) one can see that the concentration dependence of the overall degree of association is expected to have a minimum for $B \lessgtr 2.85$ in agreement with our simulation results presented in Figure 6.

Approximate Mass Balance Equation for Practical Applications. As we discussed above, the analytical solution for eq 15 provides a good match with simulation results (Figures 5 and 6). At the same time practical use of this equation can be somewhat complicated as it requires knowledge of several association constants $K_{\text {ring, } i}, K_{\text {chain }}$. The fact that we are dealing with supramolecular polymers, i.e., with flexible oligomers capable of association via orientationally specific reversible bond between end groups allows some simplifications to the equation.

Normally experimental techniques (such as infrared spectroscopy, NMR, etc.) permit measuring the overall degree of association in the system. (1) If one can measure the degree of association at very low concentration, then one can determine the (monomolecular) ring fraction as the two coincide under these conditions:

$$
\left.p\right|_{\mathrm{c} \rightarrow \mathrm{o}} \approx f_{\mathrm{r}}=\frac{\left[\mathrm{R}_{1}\right]}{[\mathrm{O}]}
$$

Knowing concentration of monomolecular rings $\left(\left[\mathrm{R}_{1}\right]=\left.[\mathrm{O}] p\right|_{\mathrm{c} \rightarrow \mathrm{o}}\right)$ and nonassociated oligomers $\left(\left[\mathrm{C}_{1}\right]=\left.(1-p)\right|_{\mathrm{c} \rightarrow \mathrm{o}}[\mathrm{O}]\right)$, one can obtain the monomolecular ring association constant:

$$
K_{\text {ring, } 1}=\frac{\left[\mathrm{R}_{1}\right]}{\left[\mathrm{C}_{1}\right]}=\left.\frac{p}{1-p}\right|_{\mathrm{c} \rightarrow \mathrm{o}}
$$

Taking into account that $K_{\text {ring, } 1}$ does not change appreciably with concentration, this gives us the association constant for monomolecular ring formation at any concentration.

(2) If one repeats measurements for the degree of association at sufficiently high oligomer concentration, one would be able to estimate the chain growth equilibrium constant $K_{\text {chain. }}$. Indeed at high concentrations the overall degree of association is defined predominantly by chain formation, and we can use eq 18 as we discussed above:

$$
\left.\frac{p}{(1-p)^{2}}\right|_{\text {high } c} \approx K_{\text {chain }}[\mathrm{O}]
$$

We note that this equation can provide a slightly overestimated value for $K_{\text {chain }}$ as this equation is designed to account for total degree of association $p$. However if the oligomer concentration is high or ring fraction is low (e.g., due to high orientational specificity of reversible bonding) then the chain fraction will provide the dominant contribution to $p$ and this overestimation will not be too significant.

(3) Now if we use the experimental values for $K_{\text {ring, } 1}$ (eq 21) and $K_{\text {chain }}$ (eq 22) and assume that for all larger size rings we can use eq 16 (which is expected to work well for oligomer lengths $N>8$ ), then we can simplify the mass balance eq 15 as follows

$$
x K_{\text {ring }, 1}+\frac{1}{i} D \frac{K_{\text {chain }}}{v N^{-\alpha}} \sum_{i \geq 2}^{\infty} i^{1-\alpha} x^{i}+\frac{x}{(1-x)^{2}}=K_{\text {chain }}[\mathrm{O}]
$$

Solving this simplified mass balance equation for the chain 
degree of association, $x$, one can predict the ring and chain distributions, degree of association, molecular weight, etc. at any concentration. The only additional information that is required is the ring closure probability for the flexible spacer (i.e., the exponent $\alpha$ and prefactor $D$ ). These values can be estimated either using the predictions for Gaussian chains: ${ }^{7} \alpha$ $=-3 / 2$ and $D=[3 /(2 \pi)]^{-3 / 2} v /\left(2 l^{3}\right)$ (where $l$ is the bond length) or using the values we obtained from our simulations of chains with excluded volume: $\alpha \approx-2$ and $D \approx 1.25$. One can also use $D$ as a fitting parameter to match the experimental data, as this parameter is sensitive to spacer chemical structure and may be hard to guess a priori. We use the simplified form of the mass balance equation eq 23 to predict the overall degree of association and fraction of reversible bonds in rings and chains shown in Figures 5 and 6 (dashed curves). As seen from Figures 5 and 6 , these values are predicted so well with the simplified equation (including the minimum at intermediate concentrations) that it is barely distinguishable from the exact solution and only for small $B$. The harder test for the approximate solution is the prediction of the maximum of ring fraction for short oligomers. As is seen from Figure 5b inset, it works rather well: even though the ring fraction is slightly overestimated the maximum in ring fraction is clearly seen and occurs at the same concentration as the simulation results. As we discuss below, a very good agreement was also obtained between the analytical solution of the simplified mass balance equation, eq 23 and simulation results for the weight-average molecular weight. These trial results give us reason to believe that this approximate equation can work reasonably well in practical applications to real experimental systems.

Ring-Chain Crossover. As we discussed above, at low concentration rings and in particular monomolecular rings are the most common architecture of associating oligomers. As the concentration increases chains start to assemble and become dominant at high concentration. The concentration at which crossover from mostly rings to mostly chains occurs can be considered as the ring-chain crossover concentration. As we discussed in our previous paper, ${ }^{29}$ this is not a true phase transition unless we are dealing with infinitely high energy of association. ${ }^{16}$ Nonetheless, the physical properties of the system (such as molecular weight or viscosity) is evidently different in the chain-dominated and ring-dominated regimes, so that the determination of this concentration can be rather useful. One can consider several different criteria for determining the ringchain crossover concentration: e.g., as the concentration where the amount of polymer in chains (including or excluding unassociated oligomers) and rings is the same ${ }^{23}$ or as the concentration where the fraction of reversible bonds in rings and chains is the same (see Figure 5). ${ }^{29}$ Since the latter is the most straightforward from the mathematical point of view, we will use the latter definition for the ring-chain crossover concentration. Figure 7 shows the oligomer number density $n_{\mathrm{rc}}$ corresponding to the ring-chain crossover as a function of oligomer length for a range of $B$ values. For flexible complexes $(B=0) n_{\mathrm{rc}}$ steadily decreases with an increase of oligomer length $\left(\sim N^{-2.2}\right)$ forming practically a straight line in the logarithmic scale of the plot. As we discussed in our previous paper, ${ }^{29}$ this dependence is somewhat similar to that for the oligomer overlap number density $n_{o} \sim R_{\mathrm{g}}^{-3} \sim N^{-3 v} \approx N^{-1.8}$ (for chains with excluded volume), but occurs at lower oligomer density. The shorter the oligomer, the larger the probability of finding its end groups within a bonding distance and therefore the larger is the fraction of rings formed (when there is no orientational limitations, i.e., $B=0$ ). As a result the crossover

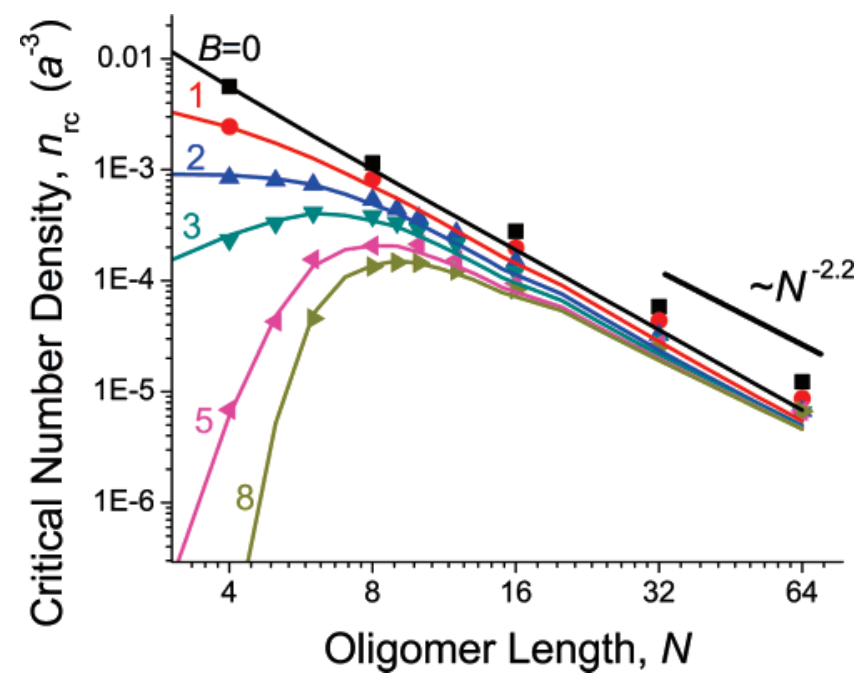

Figure 7. Critical number density $n_{\mathrm{rc}}$ corresponding to the ring-chain crossover as a function of oligomer length $N$ for different rigidity constants $B$. Simulation results are shown as symbols, while the curves are the solutions of the complete mass balance equation (eq 15).

from rings to chains occurs at a higher number density for short oligomers compared to long oligomers.

For a higher orientational specificity of reversible bonding the situation is different (Figure 7). The probability to find two ends of oligomer within a bonding distance is still the largest for the short oligomers, but the orientational limitations for reversible bonding are also the largest (especially for the monomolecular rings that dominate at low concentrations). This causes a decrease in the small ring population as $B$ increases. In particular the monomolecular ring population (see the ring distribution, Figure S3, in Supporting Information) is considerably suppressed, as the entropic loss for ring closure via an orientationally specific reversible bond $\Delta S_{\text {ring, } 1}$ increases nearly linearly with $B$ (Figure 4 ). As a result of the suppression of the (monomolecular) ring population for higher $B$ values, the crossover from rings to chains occurs at a lower oligomer density, $n_{\mathrm{rc}}$ (Figure 7). The decrease in $n_{\mathrm{rc}}$ is especially noticeable for shorter oligomer lengths where it can change by several orders of magnitude with an increase of $B$. For longer oligomers, this effect is less pronounced as in the limit of very large $N$ the entropic loss for ring closure via an orientationally specific reversible bond $\Delta S_{\text {ring, } i}$ becomes comparable to that for chains $\Delta S_{\text {bend }}$ and it increases logarithmically with $B$ (eq 9). As a result, for very long oligomers, $n_{\mathrm{rc}}$ is only slightly smaller than that for the flexible case. Thus, for orientationally specific reversible associations the ring-chain crossover occurs at lower concentrations for very short oligomers compared to the longer ones and $n_{\mathrm{rc}}$ exhibits a maximum at intermediate oligomer lengths. The position of the maximum shifts to a longer oligomer length as $B$ increases. For most of the cases considered the maximum in $n_{\mathrm{rc}}$ is achieved for $N \simeq 8$, so that for $N<8$ the orientational specificity of association has a strong effect on $n_{\mathrm{rc}}$, while for $N \geq 8, n_{\mathrm{rc}}$ is only somewhat smaller than that for flexible complexes. We note that the analytical solution of the mass balance equation (eq 15) matches the simulation data very well (Figure 7). Only small deviations can be noticed for longer oligomer lengths possibly due to somewhat worse statistics for single ring simulations leading to an overestimation of the entropic loss for ring closure.

Molecular Weight. As we discussed above, with an increase of the polymer concentration the overall degree of association increases. The molecular weight follows the same tendency, as is seen from Figure 8. 


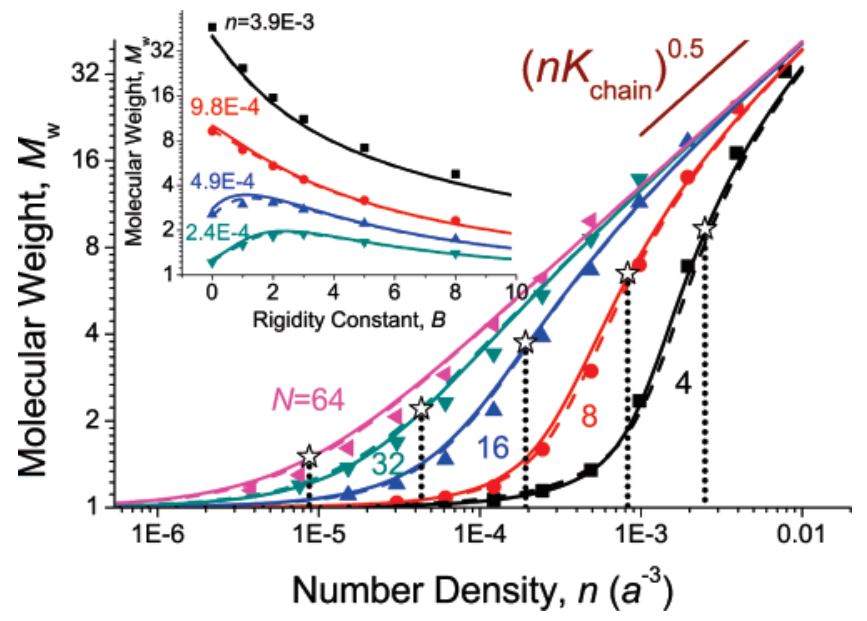

Figure 8. Weight-average molecular weight $M_{\mathrm{w}}$ (in units of oligomer weight) as a function of oligomer number density for $B=1$ and various oligomer lengths $N$. Vertical dotted lines with stars indicate the critical number density $n_{\mathrm{rc}}$ corresponding to the ring-chain crossover for each oligomer length. The inset shows the molecular weight dependence on the rigidity constant $B$ at various oligomer number densities (in units of $a^{3}$ ) for $N=8$. Simulation results are shown as symbols, while the solid and dashed curves are the solutions of the complete (eq 15) and approximate (eq 23) mass balance equation.

Comparing the weight-average molecular weight $M_{\mathrm{w}}$ (in units of oligomer weight) for different oligomer lengths at $B=1$ one can see that a considerable increase in $M_{\mathrm{w}}$ occurs at the concentration when chain association starts to increase (cf. with Figure 5a). This occurs at somewhat lower oligomer density compared to the ring-chain crossover $n_{\mathrm{rc}}$. Since the latter decreases with oligomer length (as is seen from Figure 7) the increase in $M_{\mathrm{w}}$ for longer oligomers also starts at lower polymer density.

With an increase of orientational specificity of reversible bonding the average degree of association decreases (as is seen in Figure 6) following universal dependence on $n K_{\text {chain }}$ (eq 18). As a result the weight-average molecular weight changes correspondingly, following $\left(n K_{\text {chain }}\right)^{1 / 2}$ scaling dependence in the chain-dominated regime. ${ }^{23,33,35,36}$ However in the ring-dominated regime the behavior of $M_{\mathrm{w}}$ is less universal when in some cases an increase in orientational specificity can lead to a somewhat unexpected increase in the molecular weight, as is seen for the two lower concentrations shown in the inset of Figure 8. The reason for this increase in $M_{\mathrm{w}}$ is the shift in the ring-chain equilibrium toward chain formation with an increase in $B$. As is seen from Figure 7, the ring-chain crossover shifts to lower number densities as the orientational specificity increases. As a result chain association increases as $B$ increases and the molecular weight follows the same trend. We note that except for the cases when the change in $B$ causes the ring-chain crossover to occur, the molecular weight will always decrease with an increase in orientational specificity of bonding. As is seen from Figure 8, the numerical solutions of the complete (eq 15, solid lines) and approximate mass balance equation (eq 23 , dashed lines) agree with simulation data very well, including the prediction of the maximum of the molecular weight. In the next section we will use our analytical model to predict the ring-chain crossover and molecular weight behavior for different energies of association.

Influence of the Energy of Association (Temperature). So far we have considered the influence of orientational specificity of reversible bonding on oligomer self-assembly at a fixed energy of association $\Delta E_{\text {assoc. }}$. For a different energy of association (or for the same $\Delta E_{\text {assoc }}$ but at a different temperature), the contributions of enthalpic gain and entropic losses for chain and ring formation will be different (eqs 7 and 12). As a result the behavior of all the above-mentioned properties, such as the degree of association, ring-chain crossover, molecular weight, etc. will be altered. We note that in our current approach an increase in temperature $T$ is equivalent to a decrease in the energy of association $\Delta E_{\text {assoc }}$ since the volume exclusion is enforced only by the space limitations on positions of monomers in the BFM and does not involve consideration of any interaction potentials. In reality, a change of temperature may influence volume interactions between spacers or end groups in a different manner, so some additional effects of demixing may be present.

The critical ring-chain crossover temperature calculated by numerically solving of the mass balance eq 15 is depicted in Figure 9, where $k T / \Delta E_{\text {assoc }}$ is plotted vs $n$ for two oligomer lengths $N=4$ and $N=8$ for a range of different orientational specificities of complexation. As is seen $k T / \Delta E_{\text {assoc }}$ has dramatic effect on the ring-chain crossover especially for large $B$ values and shorter oligomers. In this case the increase in $n_{\mathrm{rc}}$ can reach several orders of magnitude (Figure 9a). With a decrease of temperature the contribution of enthalpic gain increases relative to the temperature-independent entropic losses. As a result
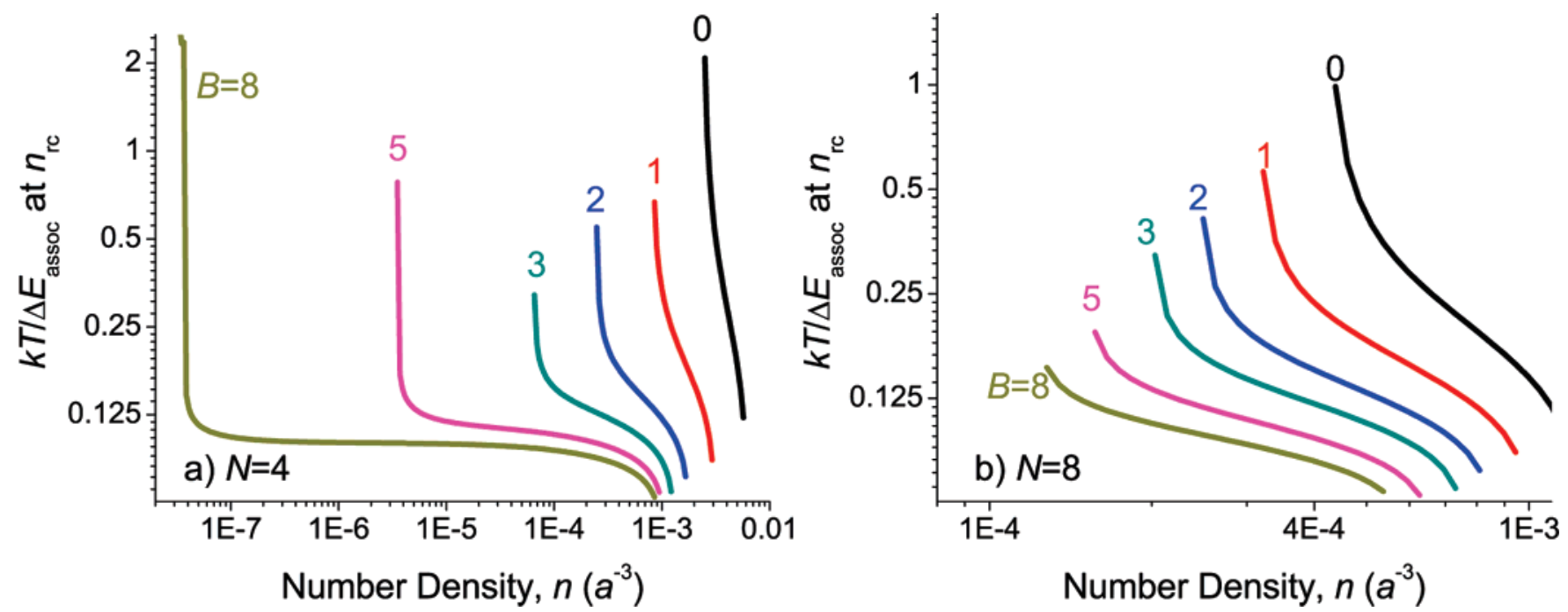

Figure 9. Numerical solutions of eq 15 for the critical $k T / \Delta E_{\text {assoc }}$ at which ring-chain crossover occurs vs oligomer number density $n$ at different $B$ values and oligomer length $N=4$ (a) and $N=8$ (b). The area below/above each curve corresponds to the ring/chain-dominated regime. 


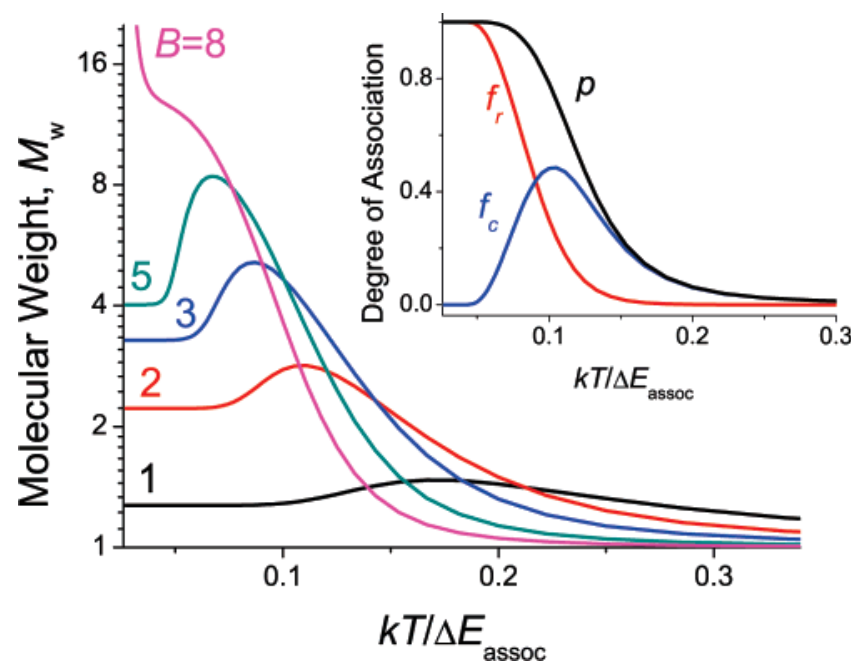

Figure 10. Numerical solutions of eq 15 for the weight-average molecular weight (in units of oligomer weight) as a function of $k T /$ $\Delta E_{\text {assoc }}$ for $N=4, n=4.8 \times 10^{-4} a^{-3}$ for various rigidity constants $B$. The inset shows the total degree of association $p$ and the fraction of reversible bonds in rings, $f_{\mathrm{r}}$, and chains, $f_{\mathrm{c}}$, for $B=5$.

formation of reversible bonds with entropically more costly orientations will become possible enhancing formation of rings (in particular monomolecular rings). This will lead to a shift of the ring-chain crossover to higher concentrations. This effect is especially strong for shorter oligomers for which $\Delta S_{\text {ring, } 1}$ is the largest (see Figure 4 and Table S1 in the Supporting Information). For longer oligomer lengths or smaller $B$ values this shift is not as significant and occurs at higher concentrations (see Figure 9b for $N=8$ and Supporting Information for other chain lengths). As is seen from Figure 9 at constant $k T / \Delta E_{\text {assoc }}$ the ring-chain crossover occurs at lower concentrations for larger $B$ values. Since lower oligomer density always favors monomolecular ring formation, below a certain concentration, (which decreases with an increase in $B$ ) rings always dominate. ${ }^{23}$

The smaller degree of association with decreasing association energy $\Delta E_{\text {assoc }}$ or increasing temperature results in the systematic decrease in molecular weight (calculated in units of oligomer weight) in the chain-dominated regime, i.e., at high concentrations or high $B$ (Figure 10).

At lower oligomer densities in the ring-dominated regime the molecular weight dependence becomes more intriguing. Indeed, as is seen from Figure 10 with an increase in $k T / \Delta E_{\text {assoc, }}$, the molecular weight actually increases for a range of $B$ values $(B$ $=1,2,3,5$, in Figure 10) until it reaches maximum at some intermediate temperature. This initial increase in the molecular weight is attributed to formation of chains. As the enthalpy of association becomes insufficient to compensate for entropic penalty for ring formation the equilibrium shifts toward chain association leading to the ring-chain crossover at somewhat higher temperature (Figure 10, inset) A further increase in temperature $k T / \Delta E_{\text {assoc }}$ results in the decrease of $M_{\mathrm{w}}$, as the enthalpic gain becomes insufficient to compensate for the entropic loss, so any association becomes less favorable. The larger the orientational specificity of association the lower is the ring-chain crossover temperature (Figure 9) and the lower the temperature corresponding to the maximum of the molecular weight (Figure 10).

The temperature $\left(k T / \Delta E_{\text {assoc }}\right)$ corresponding to the maximum of the average molecular weight $M_{\mathrm{w}}$ together with the absolute value of the molecular weight (in units of oligomer weight) at its maximum are plotted in Figure 11 for $N=4$ and different values of $B$. For rather flexible complexes $(B \lesssim 2$ for $N=4$ )
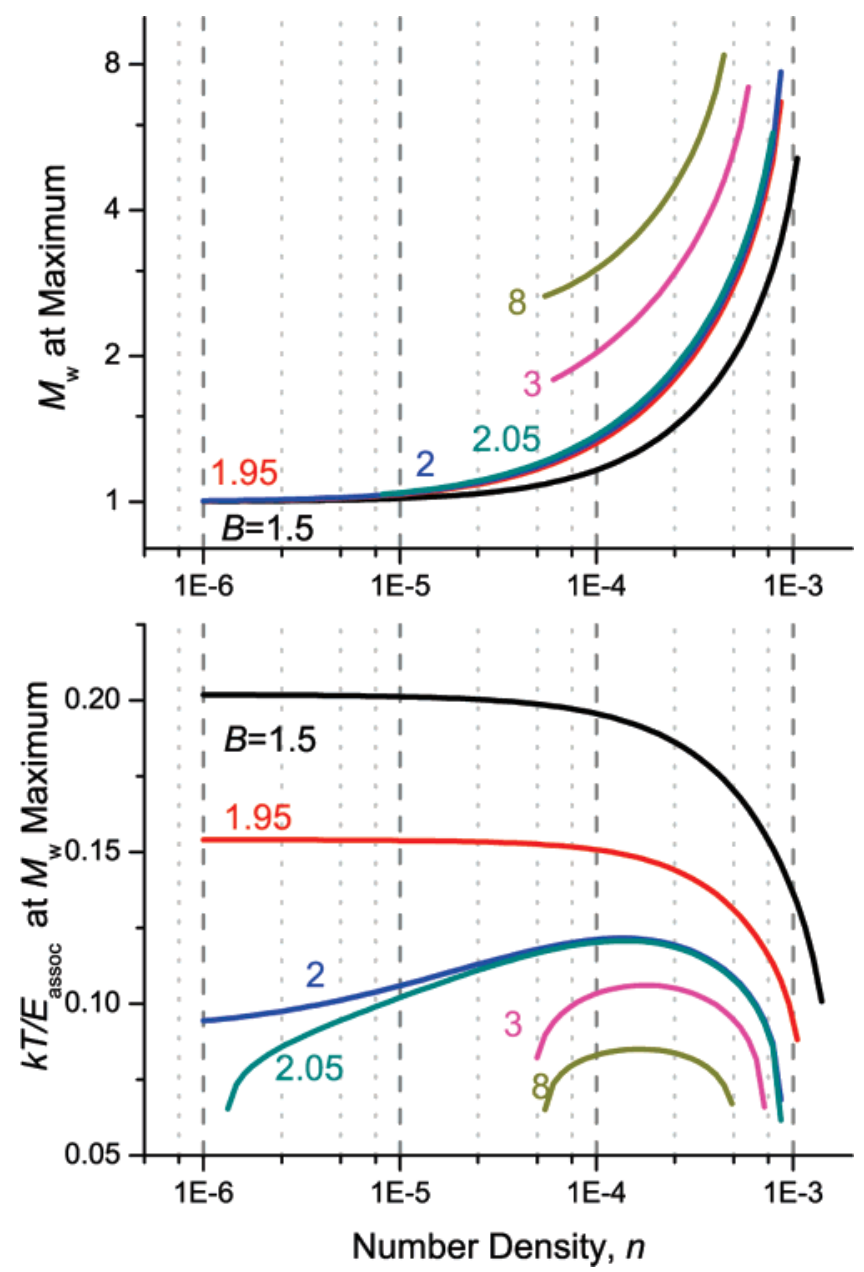

Figure 11. Numerical solutions of eq 15 for the absolute value of (weight-average) molecular weight $M_{\mathrm{w}}$ (in units of oligomer weight) (a) and $k T / \Delta E_{\text {assoc }}$ (b) at which the maximum in $M_{\mathrm{w}}$ is achieved vs oligomer number density $n$ at different $B$ values and oligomer length $N=4$.

the temperature corresponding to the maximum value of the molecular weight abruptly increases and stabilizes at some plateau level as the oligomer concentration decreases. The corresponding molecular weight sharply decreases with a decrease of $n$ and levels off at about 1. The implications of such behavior is that the smaller the oligomer concentration the more favorable rings are, so the redistribution of oligomers from rings to chains occur at increasingly higher temperature and lower average molecular weight until the limit of $M_{\mathrm{w}} \approx 1$ is reached. In this case the maximum of molecular weight corresponds to opening of a monomolecular ring. For supramolecular polymers with larger orientational specificity the behavior of the molecular weight is more complex (Figure 11). With the decrease of concentration, the temperature at which the molecular weight reaches a maximum increases similar to that for smaller $B$ values. However instead of reaching a plateau, the temperature starts to decline quickly at lower oligomer concentrations. Below some concentration no maximum in molecular weight can be observed at all. This peculiar behavior at lower $n$ is caused by the strong restrictions on formation of monomolecular rings for short oligomers with high orientational specificity of association. As a result below the concentration where bimolecular complexes becomes entropically unfavorable only a small fraction of monomolecular rings can form. Consequently no noticeable redistribution between monomolecular rings and unreacted oligomers could occur and no maximum in molecular weight could be observed. For longer 


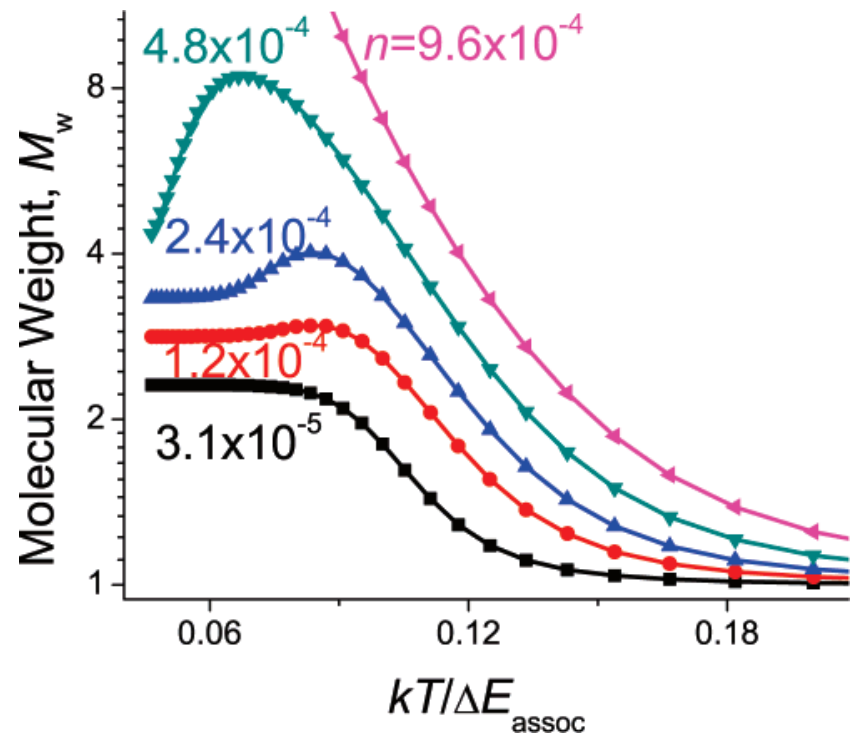

Figure 12. Numerical solutions of eq 15 for the weight-average molecular weight (in units of oligomer length $N$ ) as a function of $k T /$ $\Delta E_{\text {assoc }}$ for $N=4$ and $B=5$ at different number densities $n$ (in units of $a^{3}$ ).

oligomers formation of monomolecular rings is less restricted than for the shorter oligomers, so that the overall temperature dependence of the molecular weight (at least for $N \geq 8$ ) is rather similar to that observed at small $B$ for $N=4$, i.e. an increase and leveling off of the temperature corresponding to the maximum in $M_{\mathrm{w}}$ and corresponding decrease in the absolute value of $M_{\mathrm{w}}$ tending toward unity. In general, for any oligomer length the larger is $B$ the larger is the penalty for forming rings, which triggers redistribution of oligomers from rings to chains at lower temperatures and therefore at larger absolute values of the molecular weight.

This feature of supramolecular polymers could not be anticipated by the preceding analytical/simulation approaches, but it was observed experimentally for metal-ligand ${ }^{20}$ and reversible bonded supramolecular polymers. ${ }^{5,24}$ In both cases the orientational specificity of the complexes is appreciable: in the metal-ligand case, coordination bonds require a specific orientation of two oligomers in the vicinity of the metal, whereas in the hydrogen-bonded case the spacer was too short to form a monomolecular rings, so the bimolecular complexes dominated among rings. A similar situation is seen in our calculations (Figures 10 and 11$)$ for short spacers $(N=4)$ and larger rigidities $(B \gtrsim 2)$. For $B=5$ shown in Figure $10 K_{\text {ring, } 1}$ is more than 1

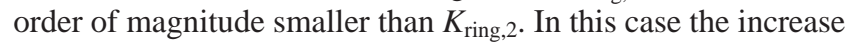
in temperature results in a 2-fold increase in molecular weight, which is consistent with the a 1.35 times increase in reduced viscosity observed in metal-ligand supramolecular polymers of stoichiometric composition ${ }^{20}$ and 4 times increase in specific viscosity of hydrogen-bonded supramolecular polymers. ${ }^{5,24} \mathrm{We}$ note that observed increase in viscosity can also be attributed to the change in the chain architecture from rings to chains. On the basis of ${ }^{1} \mathrm{H}$ NMR data, the increase in viscosity is accompanied by a $4-5$ times increase in chain population ${ }^{20,24}$ which agrees with our results (for $B=5$ ) of about a 5 times increase in chain fraction upon temperature increase (Figure 10, inset). This confirms that an increase in $M_{\mathrm{w}}$ is due to shift of the equilibrium from rings to chains.

As we can see from Figure 11 oligomer concentration also plays important role in the temperature dependence of molecular weight. A decrease in the concentration for constant $B(B=5)$ is considered in Figure 12. At the highest concentration the system is in the chain-dominated regime for the whole $k T / \Delta E_{\text {assoc }}$ range considered, so that the molecular weight steadily decreases as the temperature increases. At somewhat lower concentration the system is in the ring-dominated regime at low temperature, so with temperature increase chain formation becomes more favorable (Figure 10, inset) and the molecular weight increases. At some higher temperature the entropic loss for association overcomes the enthalpic gain resulting in the decrease in $M_{\mathrm{w}}$. The larger is the oligomer concentration the higher is the absolute value of the molecular weight at its maximum. As the oligomer density decreases the position of the maximum in $M_{\mathrm{w}}$ shifts to higher $k T / \Delta E_{\text {assoc }}$, reaches a maximum and then slightly decreases until it reaches a concentration below which the molecular weight has no maximum because large rings are unfavorable and small rings are unlikely due to their high strain for ring closure. As we discussed above, the temperature dependence of the specific viscosity for one of the hydrogenbonded supramolecular complexes studied in ref 24 exhibits a maximum followed by a decrease in viscosity at higher temperature. The same supramolecular polymer at lower concentration shows an increase in the specific viscosity, which appears to reach a maximum at a considerably higher temperature (which corresponded to the upper range of experimental measurements so the anticipated decrease in $M_{\mathrm{w}}$ at even higher temperatures was impossible to follow). That is exactly what we expect to happen in a certain concentration range based on our model (Figures 11 and 12): the lower the concentration is the stronger is the tendency to form rings, and it will take a higher temperature to make chain formation favorable, i.e., to achieve the maximum in the molecular weight (Figure 12). This trend is qualitatively similar to what was observed for decreasing $B$ in the main plot of Figure 10. Both a decrease in oligomer concentration and a decrease in orientational specificity favor small ring formation (if orientational specificity and oligomer length allows), leading to the increase of temperatures corresponding to ring-chain crossover and the maximum of the molecular weight.

In general to observe experimentally an increase in $M_{\mathrm{w}}$ or viscosity with a temperature increase the change in molecular weight should be significant. This can be achieved for the systems with ring-chain crossover occurring at relatively high concentrations and low temperatures. The requirements for designing such supramolecular system are the following: use a flexible spacer of a rather short length and supramolecular associating units of intermediate-to-high orientational-specificity. In such case bimolecular or (even higher order) rings will be preferable at low temperature, and a temperature increase will shift the equilibrium to chains of even higher degree of association, so that molecular weight will noticeably increase. Alternatively the chemical structure of associating oligomers can be tuned to preferentially form large rings at low-tointermediate concentrations and temperatures followed by chains at larger concentrations or temperatures. For rigid spacers ring formation will be greatly diminished (unless they are designed to form rings) and no noticeable increase in molecular weight can be expected with increasing temperature. Similarly, for flexible spacers with weakly orientationally specific associating groups, a high molecular weight at its maximum is also hard to achieve since single rings will persist until very high temperatures where any associations are not favorable.

The degree of association in supramolecular polymer system can be measured by infrared spectroscopy or NMR which permit detection of the relative fraction of associated and nonassociated groups. These measurements could be performed for flexible 
spacers (such as PEO) of different length as a function of concentration. On the basis of these measurements, the validity of eq 18 in a relatively high concentration range can be tested. Additionally NMR measurements may allow simultaneous detection of the concentration of smallest size rings and the rest of the associating species. ${ }^{5}$ Since small rings are the dominant species at low concentrations, comparison of the concentration of rings and the rest of the associating species at different concentrations would allow one to estimate the ring-chain crossover concentration (which would be rather close to $n_{\mathrm{rc}}$ ). Repeating such measurements for different spacer length and possibly different associating groups would allow a comparison of the experimental results with our predictions shown in Figure 7 (including the $N^{-2.2}$ scaling dependence expected for longer spacers), while measurements performed at different temperatures can be compared with the temperature dependence of the crossover concentration plotted in Figure 9. Determination of the average molecular weight experimentally is more challenging, as many traditional techniques (e.g., static light scattering with an extrapolation to zero concentration) would induce a change in the equilibrium. This problem can be avoided for association complexes with a high energy of association $\Delta E_{\text {assoc }}$ if the system is allowed to equilibrate at some high temperature and then quickly quenched to low temperature when dissociation will be highly unfavorable. Another less direct way of measurements of molecular weight is by using zero-angle light scattering or via viscosity measurements. ${ }^{5,24}$ The experimental values for molecular weight measured at different concentrations and temperatures can be compared with our predictions shown in Figures 6 and 10-12. As we discussed above, some of the reported viscosity measurements, ${ }^{5,24}$ show good potential for such comparisons. We also plan to expand our approach to the prediction of dynamic properties of supramolecular polymers including viscosity.

\section{Conclusions}

We have applied Monte Carlo simulations using the bond fluctuation model to study the influence of orientational specificity of reversible association on the self-assembly and properties of supramolecular polymers. We have studied linear flexible oligomers consisting of $N-2$ flexible spacer monomers and two terminal groups, one a donor and the other an acceptor for reversible association, which can lead to formation of rings and chains. The orientational specificity of reversible association was implemented in our simulations by applying a weighting factor for formation of a reversible bond at a given angle $\theta$ : $P(\theta)=\exp [-B(1-\cos \theta)]$ with $B$ being defined as a rigidity constant. On the basis of simulation results we calculated the equilibrium constants for chain growth $\left(K_{\text {chain }}\right)$ and ring closure $\left(K_{\text {ring }, i}\right)$ via a reversible bond. We found that the latter strongly varies with oligomer length $N$, especially for short oligomers. For longer oligomers $N \geq N_{\max } \approx 15$ the entropic loss originating from orientational limitations becomes comparable to that for chains (Figure 4). In this case the equilibrium constant for ring closure $\left(K_{\text {ring, }, i}\right)$ can be related to $K_{\text {chain. }}{ }^{16,17}$ This observation allowed us to suggest an approximate mass balance equation (eq 23) which can be used to predict the properties of supramolecular polymers and analyze experimental data. To this end all that is necessary to know is the experimental degree of association at high and low concentrations, and to have some idea about the end-to-end distribution for the oligomer (e.g., Gaussian-like or excluded volume-like behavior). This will provide an estimate of the equilibrium constants for chain growth $K_{\text {chain }}$ and smallest ring closure $K_{\text {ring, } 1}$ which then can be plugged into the approximate mass balance equation (eq 23). Solving this equation for the chain degree of association allows one to obtain the chain and ring distribution, degree of association, and molecular weight and to estimate the ring-to chain crossover concentration.

We found that the orientational specificity of reversible bonding significantly affects the overall degree of association $p$ as well as the fraction of reversible bonds in rings $f_{\mathrm{r}}$ and chains $f_{\mathrm{c}}$. At low concentration, the overall degree of association is defined by monomolecular ring formation. For small $B$ values, ring formation is favorable and the overall degree of association is relatively high even at low concentrations (Figure 5). At the same time for larger $B$, the entropic penalty associated with the angle limitations for ring closure (especially for short oligomers) is very high, so monomolecular rings are not so likely and the degree of association is rather small at low concentrations (Figure 5). At higher concentrations formation of bimolecular rings becomes possible and the ring degree of association may increase if $K_{\text {ring, } 2}>K_{\text {ring, } 1}$. However, in most of the cases (for longer oligomers or smaller $B$ ) an increase in oligomer density leads normally to enhancement in chain degree of association, which dominates at high concentrations. As a result, the overall degree of association increases in a uniform manner as a function of $n K_{\text {chain }}$ (eq 18) at large oligomer concentration ${ }^{35}$ and it decreases with $B$ (Figure 6). The weight-average molecular weight follows the same pattern as the degree of association: in the chain-dominated regime it increases following $\left(n K_{\text {chain }}\right)^{1 / 2}$

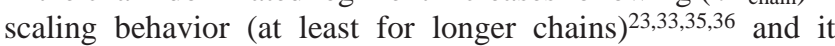
decreases with an increase of orientational specificity of association. The abrupt increase in $M_{\mathrm{w}}$ occurs somewhat in advance to the ring-chain crossover when formation of chains becomes favorable. As the ring-chain crossover shifts to lower oligomer density with an increase in oligomer length or in increase in $B$, the molecular weight starts to increase at smaller oligomer densities as well. Our analytical predictions based on numerical solution of the complete or approximate mass balance equation are shown to be very successful in predicting the degree of association as well as the molecular weight and ring-chain crossover.

We have also considered the influence of oligomer length and orientational specificity of reversible association on the ring-chain crossover, which we define as the oligomer density $n_{\mathrm{rc}}$ at which the number of reversible bonds in rings and chains are equal to each other. We found that for flexible reversible associations the oligomer number density corresponding to the ring-chain crossover $n_{\mathrm{rc}}$ steadily decreases with oligomer length $N$. With an increase in $B$, the entropic penalty associated with orientational specificity of reversible bonding comes into play which results in a further decrease in the ring-chain crossover oligomer density (Figure 7). This effect is especially strong for short oligomers, for which $n_{\mathrm{rc}}$ decreases by several orders of magnitude, while for longer oligomers the decrease is rather marginal. As a result $n_{\mathrm{rc}}$ first increases with increasing oligomer length $N$, then exhibits a maximum at intermediate $N$ and finally decreases at large $N$. The larger the orientational specificity of reversible bonding the larger the amplitude of the effect.

Using our analytical approach, which reproduces all simulation data very well, we have also analyzed the influence of temperature on the ring-chain crossover and molecular weight behavior. In our approach, an increase in the energy of association is equivalent to the decrease of temperature as long as it leads to the same $\Delta E_{\text {assoc }} /(k T)$ ratio. A decrease in temperature implies enhancement of the enthalpic gain of reversible bond formation, which allows formation of smaller size rings with less entropically favorable bonding orientations. 
As a result the ring-chain crossover shifts to larger concentrations with a temperature decrease. This effect is especially strong for large $B$ values and shorter oligomers for which the increase in the number density corresponding to ring-chain crossover can reach several orders of magnitude. In general the higher the orientational specificity of bonding the lower is the temperature (higher the $\Delta E_{\text {assoc }}$ ) at which the crossover occurs (Figure 9).

The implications of these changes in ring-chain crossover for the molecular weight behavior as a function of energy/ temperature are found to be the following: In the chaindominated regime (i.e., at sufficiently high concentrations) an increase of temperature simply results in the decrease in molecular weight (and by extension in the viscosity, as is often seen experimentally ${ }^{25,37}$ ). However in the ring-dominated regime (i.e., at lower concentrations) a temperature increase may lead to an increase in the molecular weight due to shift of the equilibrium from rings to chains. This prediction agrees well with recent experimental observations for metallo-supramolecular and hydrogen-bonded polymers. ${ }^{20,24}$ The position of the maximum of the $M_{\mathrm{w}}$ shifts to higher temperatures and its absolute value decreases with a decrease of concentration. (Figures 11 and 12) The latter was experimentally observed for hydrogen-bonded supramolecular polymers. ${ }^{24}$ At higher temperature the enthalpic gain becomes insufficient to compensate for entropic loss and $M_{\mathrm{w}}$ starts to decrease. An increase in orientational specificity $B$ inhibits ring formation (especially monomolecular rings from short oligomers) and decreases the ring-chain crossover temperature leading also to the decrease of the temperature at which the maximum of the $M_{\mathrm{w}}$ is reached and an increase in the absolute value of $M_{\mathrm{w}}$ (Figures 10,11). Therefore, to observe experimentally an increase in $M_{\mathrm{w}}$ or viscosity with a temperature increase it is preferable to use associating units of intermediate-to-high orientational-specificity (and select a spacer which prefers formation of rings of larger sizes).

\section{Appendix}

A. Analytical Calculations in Zero Density Limit. In the zero concentration limit, the properties of the system are determined by the behavior of a single oligomer. The degree of association at zero concentration is defined by the probability of forming a monomolecular ring $p_{\mathrm{o}}$, which is connected to the concentration of chains and rings as $\left[\mathrm{C}_{1}\right]=\left(1-p_{\mathrm{o}}\right)[\mathrm{O}]$ and $\left[\mathrm{R}_{1}\right]=p_{\mathrm{o}}[\mathrm{O}]$ where $[\mathrm{O}]$ is the original concentration of oligomers. The degree of association is

$$
p=\frac{\left[\mathrm{C}_{1}\right] \times 0+\left[\mathrm{R}_{1}\right] \times 1}{[\mathrm{O}]}=p_{\mathrm{o}}
$$

while the ring fraction is

$$
w=\frac{\left[\mathrm{R}_{1}\right]}{[\mathrm{O}]}=p_{\mathrm{o}}
$$

$p_{\mathrm{o}}$ is related to the equilibrium constant for monomolecular ring formation

$$
\begin{aligned}
K_{\text {ring }, 1} & \equiv \frac{\left[\mathrm{R}_{1}\right]}{\left[\mathrm{C}_{1}\right]}=\frac{p_{\mathrm{o}}}{1-p_{\mathrm{o}}} \\
& \Longrightarrow p=p_{\mathrm{o}}=\frac{K_{\text {ring }, 1}}{1+K_{\text {ring }, 1}}
\end{aligned}
$$

With an increase in the concentration it becomes possible to form chains and rings of size two in the system. The degree of association becomes

$$
\begin{aligned}
p & =\frac{\left[\mathrm{C}_{2}\right]+\left[\mathrm{R}_{1}\right]+2\left[\mathrm{R}_{2}\right]}{\left[\mathrm{C}_{1}\right]+2\left[\mathrm{C}_{2}\right]+\left[\mathrm{R}_{1}\right]+2\left[\mathrm{R}_{2}\right]} \\
& =\frac{K_{\text {ring, } 1}+K_{\text {chain }}\left(1+2 K_{\text {ring }, 2}\right)\left[\mathrm{C}_{1}\right]}{1+K_{\text {ring }, 1}+2 K_{\text {chain }}\left(1+K_{\text {ring }, 2}\right)\left[\mathrm{C}_{1}\right]}
\end{aligned}
$$

The degree of association $p$ decreases with $\left[\mathrm{C}_{1}\right]$ if $\partial p / \partial\left[\mathrm{C}_{1}\right]<$ 0 , resulting in

$$
\frac{1+2 K_{\text {ring }, 2}}{2+2 K_{\text {ring }, 2}}<\frac{K_{\text {ring }, 1}}{1+K_{\text {ring }, 1}}
$$

or

$$
1+2 K_{\text {ring, } 2}<K_{\text {ring, } 1}
$$

The ring fraction $w$ becomes

$$
\begin{aligned}
w & =\frac{\left[\mathrm{R}_{1}\right]+2\left[\mathrm{R}_{2}\right]}{\left[\mathrm{C}_{1}\right]+2\left[\mathrm{C}_{2}\right]+\left[\mathrm{R}_{1}\right]+2\left[\mathrm{R}_{2}\right]} \\
& =\frac{K_{\text {ring }, 1}+2 K_{\text {chain }} K_{\text {ring }, 2}\left[\mathrm{C}_{1}\right]}{1+K_{\text {ring }, 1}+2 K_{\text {chain }}\left(1+K_{\text {ring }, 2}\right)\left[\mathrm{C}_{1}\right]}
\end{aligned}
$$

The ring fraction increases with $\left[\mathrm{C}_{1}\right]$ if $\partial w / \partial\left[\mathrm{C}_{1}\right]>0$ leading to

$$
\frac{K_{\text {ring, } 2}}{1+K_{\text {ring }, 2}}>\frac{K_{\text {ring, } 1}}{1+K_{\text {ring, }, 1}}
$$

or

$$
K_{\text {ring, }, 2}>K_{\text {ring, } 1}
$$

Since the degree of association will eventually become $p=1$ in the high concentration limit, a minimum in $p$ must exist when the condition A7 is satisfied. On the other hand, the ring fraction will decrease to $w=0$ in the high concentration limit and a maximum in $w$ must exist when the condition A10 is satisfied.

A.1. Gaussian Chain Approximation. For a Gaussian chain of $N$ monomer units, the probability for the two ends to meet is given by $A N^{-3 / 2}$ where $A$ is a normalization constant. Considering the energy decrease $\Delta E_{\text {assoc }}$ for forming of a flexible reversible bond $(B=0)$, the equilibrium constant for the monomolecular ring closure is given by

$$
K_{\text {ring, }, 1}^{\mathrm{flex}}=A N^{-3 / 2} \mathrm{e}^{\Delta E_{\text {assoc }} / k T}
$$

We can apply similar consideration to association of two oligomers. However, there are two ways to form a linear chain of size two and only one way to form a ring of size two out of two oligomers. Therefore, the equilibrium constant for formation of flexible bimolecular ring $(B=0)$ is given by

$$
K_{\text {ring, }, 2}^{\mathrm{flex}}=\frac{A(2 N)^{-3 / 2} \mathrm{e}^{\Delta E / k T}}{2}=2^{-5 / 2} K_{\text {ring }, 1}^{\mathrm{flex}}
$$

The condition A7 becomes

$$
K_{\text {ring, }, 1}^{\mathrm{flex}}>\left(1-2^{-3 / 2}\right)^{-1} \approx 1.547
$$


which, using eq A4, gives the condition for the degree of association at zero concentration

$$
\left.p\right|_{\mathrm{n} \rightarrow \mathrm{o}}>\left(2-2^{-3 / 2}\right)^{-1} \approx 0.607
$$

If the degree of association is larger than 0.607 at low polymer concentrations then one can expect to observe a minimum in $p$ with an increase of concentration.

Acknowledgment. This material is based upon work supported by the National Science Foundation under Grant No. 0348302. We also express gratitude to the Case Alumni Association supported summer research of M.H. as a part of the SOURCE research initiative for undergraduates at Case Western Reserve University.

Supporting Information Available: Text giving the details of single oligomer simulations and estimated entropy loss due to orientational specificity of reversible bond, radius of gyration and aspect ratio for the chains with orientational specificity of association, as well as ring distribution and ring-chain crossover diagram for different energies of association, a table of entropic loss data, and figures showing the square radius of gyration as a function of the number of oligomers, aspect ratio for chains, ring distribution as a fraction of ring size, and numerical solutions of eq 15 . This material is available free of charge via the Internet at http:// pubs.acs.org.

\section{References and Notes}

(1) Lehn, J.-M. Supramolecular Chemistry: Concepts and Perspectives; VCH: Weinheim, Germany, 1995.

(2) Lehn, J.-M. In Supramolecular science: Where it is and where it is going; Ungaro, R., Dalcanale, E., Eds.; Kluwer: Dordrecht, Netherlands, 1999; pp 287-304.

(3) Lehn, J.-M. Science 2002, 295, 2400-2403.

(4) Prins, L. J.; Reinhoudt, D. N.; Timmerman, P. Angew. Chem., Int. Ed. 2001, 40, 2382-2426.

(5) ten Cate, A. T.; Sijbesma, R. P. Macromol. Rapid Commun. 2002, 23, 1094-1112.

(6) Dobrawa, R.; Würthner, F. J. Polym. Sci., Part A: Polym. Chem. 2005, 43, 4981-4995

(7) Jacobson, H.; Stockmayer, W. H. J. Chem. Phys. 1950, 18, 16001606.

(8) Flory, P. J.; Suter, U. W.; Mutter, M. J. Am. Chem. Soc. 1976, 98 , $5733-5739$
(9) Yamakawa, H.; Stockmayer, W. H. J. Chem. Phys. 1972, 57, $2843-$ 2854.

(10) Shimada, J.; Yamakawa, H. Macromolecules 1984, 17, 689-698.

(11) Ercolani, G.; Mandolini, L.; Mencarelli, P.; Roelens, S. J. Am. Chem. Soc. 1993, 115, 3901-3908.

(12) Kuchanov, S.; Slot, H.; Stroeks, A. Prog. Polym. Sci. 2004, 29, 563633 and references therein.

(13) Mattice, W. L. J. Am. Chem. Soc. 1980, 102, 2242-2245.

(14) Chen, Z.-R.; Claverie, J. P.; Grubbs, R. H.; Kornfield, J. A. Macromolecules 1995, 28, 2147-2154.

(15) Van Workum, K.; Douglas, J. F. Phys. Rev. E 2005, 71, 031502.

(16) Petschek, R. G.; Pfeuty, P.; Wheeler, J. C. Phys. Rev. A 1986, 34 2391-2421

(17) Corrales, L. R.; Pfeuty, P.; Wheeler, J. C. J. Chem. Phys. 1989, 90 , $5030-5055$

(18) Cates, M. E.; Candau, S. J. J. Phys.: Condens. Matter 1990, 2, 68696892.

(19) Lahn, B.; Rehahn, M. e-Polym. 2002, no. 001.

(20) Vermonden, T.; van der Gucht, J.; de Waard, P.; Marcelis, A.; Besseling, N.; Sudholter, E.; Fleer, G.; Stuart, M. Macromolecules 2003, 36, 7035-7044.

(21) Loverde, S. M.; Ermoshkin, A. V.; de la Cruz, M. O. J. Polym. Sci., Part B: Polym. Phys. 2005, 43, 796-804.

(22) Milchev, A.; Wittmer, J. P.; Landau, D. P. Phys. Rev. E 2000, 61, $2959-2966$

(23) Wittmer, J. P.; van der Schoot, P.; Milchev, A.; Barrat, J. L. J. Chem. Phys. 2000, 113, 6992-7005.

(24) Folmer, B. J. B.; Sijbesma, R. P.; Meijer, E. W. J. Am. Chem. Soc. 2001, 123, 2093-2094.

(25) Sijbesma, R. P.; Beijer, F. H.; Brunsveld, L.; Folmer, B. J. B.; Hirschberg, J. H. K. K.; Lange, R. F. M.; Lowe, J. K. L.; Meijer, E. W. Science 1997, 278, 1601-1604

(26) Carmesin, I.; Kremer, K. Macromolecules 1988, 21, 2819-2823.

(27) Deutsch, H. P.; Binder, K. J. Chem. Phys. 1991, 94, 2294-2304.

(28) Similar potentials have been used for covalent bonds in previous applications of BFM: Wittmer, J.; Paul, W.; Binder, K. Macromolecules 1992, 25, 7211-7219.

(29) Chen, C.-C.; Dormidontova, E. E. Macromolecules 2004, 37, 39053917.

(30) Wittmer, J. P.; Milchev, A.; Cates, M. E. J. Chem. Phys. 1998, 109, $834-845$.

(31) des Cloizeaux, J.; Jannink, G. Polymers in Solution: Their Modelling and Structure; Oxford University Press: New York, 1990.

(32) Porte, G. J. Chem. Phys. 1983, 87, 3541-3550.

(33) Cates, M. E. J. Phys. (Paris) 1988, 49, 1593-1600.

(34) Suter, U. W.; Höcker, H. Makromol. Chem. 1988, 189, 1603-1612.

(35) van der Schoot, P. In Supramolecular Polymers, 2nd ed.; Ciferri, A., Ed.; CRC Press: Boca Raton, FL, 2005; p 77.

(36) Wittmer, J. P.; Milchev, A.; Cates, M. E. Europhys. Lett. 1998, 41, 291-296.

(37) Kang, H.; Lin, Q.; Armentrout, R. S.; Long, T. E. Macromolecules 2002, 35, 8738-8744.

MA062146+ 